\title{
A new generation needle- and adjuvant-free trivalent plague vaccine utilizing adenovirus-5 nanoparticle platform
}

\author{
Paul B. Kilgore ${ }^{1}$, Jian Sha ${ }^{1,2 凶}$, Jourdan A. Andersson ${ }^{1}$, Vladimir L. Motin ${ }^{1,2,3,4,5}$ and Ashok K. Chopra ${ }^{1,2,4,5 凶}$
}

A plague vaccine with a fusion cassette of YscF, F1, and LcrV encoding genes in an adenovirus-5 vector (rAd5-YFV) is evaluated for efficacy and immune responses in mice. Two doses of the vaccine provides $100 \%$ protection when administered intranasally against challenge with Yersinia pestis CO92 or its isogenic F1 mutant in short- or long- term immunization in pneumonic/bubonic plague models. The corresponding protection rates drop in rAd5-LcrV monovalent vaccinated mice in plague models. The rAd5-YFV vaccine induces superior humoral, mucosal and cell-mediated immunity, with clearance of the pathogen. Immunization of mice with rAd5-YFV followed by CO92 infection dampens proinflammatory cytokines and neutrophil chemoattractant production, while increasing Th1- and Th2-cytokine responses as well as macrophage/monocyte chemo-attractants when compared to the challenge control animals. This is a first study showing complete protection of mice from pneumonic/bubonic plague with a viral vectorbased vaccine without the use of needles and the adjuvant.

npj Vaccines (2021)6:21; https://doi.org/10.1038/s41541-020-00275-3

\section{INTRODUCTION}

Yersinia pestis, the causative agent of plague, is a Tier-1 select agent and a re-emerging human pathogen ${ }^{1,2}$. There have been three plague worldwide pandemics, which were responsible for over 200 million deaths ${ }^{3}$. The most recent large outbreak of plague (2017-2018) occurred in Madagascar which resulted in $\sim 2400$ cases and $\sim 200$ deaths $^{4}$. Importantly, over $75 \%$ of the plague cases were pneumonic in this outbreak ${ }^{5}$. Traditionally, most cases of plague globally are bubonic in nature, which has a lower case-fatality rate and longer disease course than pneumonic plague $^{3}$. Pneumonic plague, on the other hand, has a shorter disease course and an almost $100 \%$ fatality rate if not treated with appropriate antibiotics within $24 \mathrm{~h}$ of symptom onset, making it an urgent public health priority $^{6}$. Indeed, Vallès et al. recently emphasized the importance of developing a clear roadmap on plague vaccines based on the current knowledge gaps ${ }^{7}$.

There are currently no Food and Drug Administration (FDA) approved vaccines to protect human population against plague ${ }^{8,9}$. However, FDA did provide "Orphan Drug" designation to the rF1-V vaccine (composed of recombinant capsular antigen F1 and a type 3 secretion system [T3SS] component [tip of the T3SS needle] and an effector, LcrV or $\mathrm{V}$ antigen) developed by DynPort Vaccine Company ${ }^{10-12}$. This vaccine has completed phase $2 \mathrm{~b}$ clinical trials (https://clinicaltrials.gov/ct2/results?cond=plague\&term $=\& \mathrm{cntr}$ $\mathrm{y}=\&$ state $=\&$ city $=\&$ dist $=$ ). Plague subunit vaccines are generally administered in the host parenterally in conjunction with an adjuvant ${ }^{13,14}$. Likewise, a live-attenuated plague vaccine, $Y$. pestis EV76 ${ }^{15}$, is used in humans outside the United States in the Former States of Soviet Union, Mongolia, and China. This vaccine strain, which lacks the pigmentation locus $(\mathrm{pgm})$ needed to acquire iron from the host, is protective against both bubonic and pneumonic plague $^{16}$. However, it is highly reactogenic and there are concerns about its safety in certain human populations such as those with hemochromatosis ${ }^{17}$.
There have been reports of naturally occurring F1-negative $Y$. pestis strains that can be as common as $10-16 \%$ in field sampling studies $^{18}$. Importantly, F1-negative $Y$. pestis strains are still fully virulent $^{19}$. Likewise, LcrV has hypervariable regions. There are at least five variants (clades) of LcrV based on the phylogenetic analysis of the conformational segment (amino acid [aa] residues 218-233) and the aa residue at position 255, from $43 Y$. pseudotuberculosis strains ${ }^{20}$. Most importantly, LcrV variants from different clades when expressed from Lactococcus lactis did not offer cross-protection during infection in vivo models with $Y$. pseudotuberculosis ${ }^{20}$. Therefore, there is always a concern that $\mathrm{rF1}$ $\mathrm{V}$ based subunit vaccines might not be fully protective against all $Y$. pestis strains, including the F1-negative and those which possess LcrV variants, that could cause an outbreak of plague ${ }^{21}$. Since $Y$. pestis has evolved from $Y$. pseudotuberculosis, plaguecausing strains with LcrV variants are expected to exist in nature 22,23 .

We have previously developed a replication-defective human adenovirus-5 (Ad5) vector-based vaccine containing three $Y$. pestis antigens and included: YscF which constitutes needle of the T3SS, the capsule protein $\mathrm{F} 1$, and the T3SS component and effector $\mathrm{LCrV}^{24}$. The vaccine was designated as rAd5-YFV. Supplementary Fig. 1 depicted amino acid sequence of the rAd5-YFV construct. The details for generating rAd5-YFV trivalent- and rAd5-LcrV monovalent-vaccines, expression of the corresponding genes encoding LcrV and YFV (as determined by SDS-PAGE and western blot analysis using LcrV antibodies, as well as ELISA by coating plates with individual YscF, F1, and LcrV antigens), were provided in our earlier paper ${ }^{24}$.

The rAd5-YFV vaccine was initially tested either as a single dose alone $\left(8.0 \times 10^{9}\right.$ virus particles [v.p] intranasally [i.n.] or intramuscularly [i.m.]) or followed by a booster of purified recombinant trivalent fusion antigen (rYFV) administered i.m. after 15 days of the first vaccine dose in a prime-boost strategy. One dose of the rAd5-YFV vaccine (delivered i.n.) offered significant protection

\footnotetext{
${ }^{1}$ Department of Microbiology \& Immunology, University of Texas Medical Branch, Galveston, TX, USA. ${ }^{2}$ Institute for Human Infections and Immunity, University of Texas Medical Branch, Galveston, TX, USA. ${ }^{3}$ Department of Pathology, University of Texas Medical Branch, Galveston, TX, USA. ${ }^{4}$ Sealy Institute for Vaccine Sciences, University of Texas Medical Branch, Galveston, TX, USA. ${ }^{5}$ Galveston National Laboratory, University of Texas Medical Branch, Galveston, TX, USA. ${ }^{\circledR}$ email: jisha@utmb.edu; achopra@utmb.edu
} 
a

\begin{tabular}{|l|l|c|c|}
\hline $\begin{array}{l}\text { Groups } \\
(\mathbf{n = 1 3})\end{array}$ & $\begin{array}{c}\text { Vaccine and } \\
\text { route of } \\
\text { delivery }\end{array}$ & $\begin{array}{c}\text { PBS or } \\
\text { Vaccine } \\
\text { doses }\end{array}$ & $\begin{array}{c}\text { Challenge organism, } \\
\text { dose, and route }\end{array}$ \\
\hline 1 & Control (i.n.) & PBS & CO92-lux $\left(100 \mathrm{LD}_{50}\right)$, i.n. \\
\hline 2 & rAd5-YFV (i.n.) & 1 & CO92-lux $\left(100 \mathrm{LD}_{50}\right)$, i.n. \\
\hline 3 & rAd5-YFV (i.n.) & 2 & CO92-lux $\left(100 \mathrm{LD}_{50}\right)$, i.n. \\
\hline
\end{tabular}

C

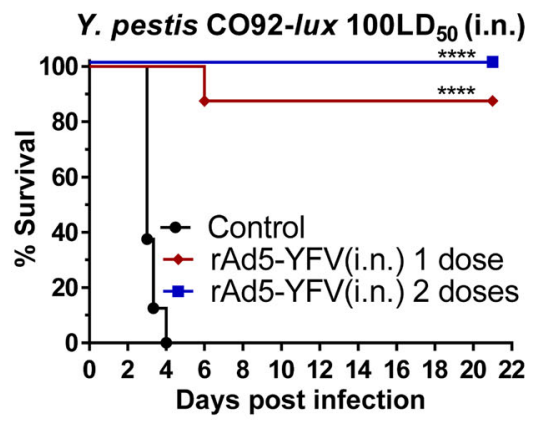

d

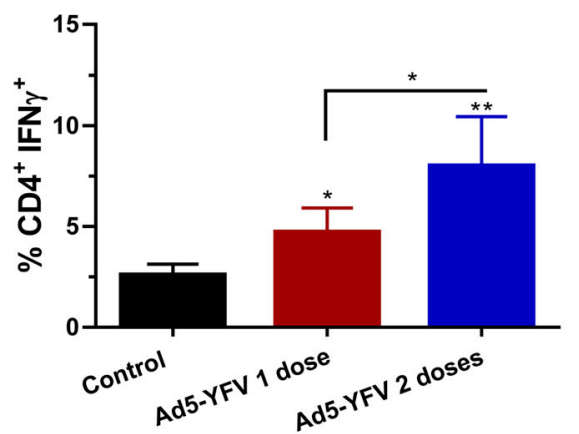

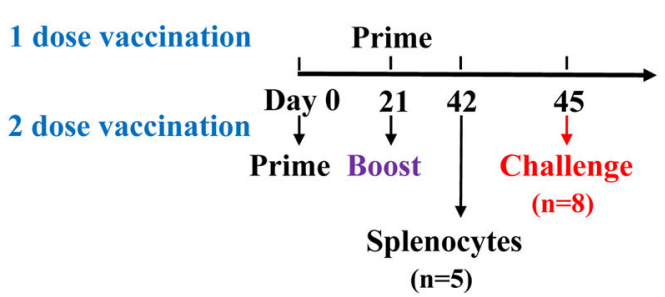

$\mathbf{e}$

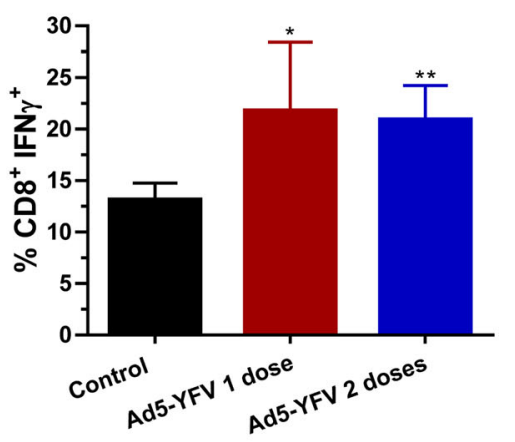

Fig. 1 Protection and immune responses in mice immunized with 1 or $\mathbf{2}$ doses of the rAd5-YFV vaccine. Female Swiss-webster mice $(n=$ 13/group) were immunized (i.n.) with either 1 or 2 doses ( 21 days apart) of rAd5-YFV vaccine $\left(1.2 \times 10^{10}\right.$ v.p.), and animals administered PBS served as controls (a). Vaccination scheme is depicted in panel b. Twenty-four days after the final vaccination, eight mice from each group were i.n. challenged with $100 \mathrm{LD}_{50}\left(5 \times 10^{4} \mathrm{CFU} / 40 \mu \mathrm{L}\right)$ of $Y$. pestis CO92-lux, and the survival of animals plotted (c). $P$ values were calculated using Kaplan-Meier analysis with log-rank (Mantel-Cox) test for animal survival. Spleens were harvested from the five remaining unchallenged mice in each group and splenocytes stimulated with PMA and lonomycin. Brefeldin A was added to prevent secretion of cytokines. Splenocytes were then stained for T-cell surface markers CD3, CD4, and CD8, followed by intracellular IFN $\gamma$ staining. Percent of $\mathrm{CD}^{+} \mathrm{IFN} \gamma^{+}$(d) and $\mathrm{CD}^{+} \mathrm{IFN}^{+}$(e) T cells were analyzed by flow cytometry and the data expressed as the arithmetic means \pm standard deviations. $P$ values were calculated using a one-way ANOVA with Tukey post-hoc test to compare multiple groups or student $t$-test to compare 1 dose of rAd5-YFV vaccine to control in panel e. Asterisks above columns represent comparison to the control group, while horizontal bars represent differences between test groups. ${ }^{*} P<0.05,{ }^{* *} P<0.01,{ }^{* * *} P<0.0001$. Two biological replicates were performed, and data plotted.

(60\%) in a pneumonic plague mouse model when challenged with $\sim 100 \mathrm{LD}_{50}$ of $Y$. pestis CO92. However, a rYFV protein boost was needed to achieve complete protection in both mouse as well as non-human primate models at much higher challenge doses of aerosolized $Y$. pestis $\mathrm{CO}^{24}$.

Recently, the World Health Organization (WHO) has released preferred target product profile (TPP) of plague vaccines ${ }^{25}$. The recommendations included a needle-free vaccine that should not be administered in more than 2 doses, and the vaccine should generate long-lasting immune responses. In this study, we evaluated our rAd5-YFV vaccine delivered in 1 or 2 doses ( 21 days apart) by the i.n. route without a boost of the rYFV fusion protein. Subsequently, we compared rAd5-LcrV monovalent and rAd5-YFV trivalent vaccines in a short-term study (challenge on day 45 of the study, 24 days [ 3 weeks] after the 2nd vaccination dose), and a long-term study (challenge on day 105 of the study, 85 days [ 12 weeks] after the 2 nd vaccination dose) for their efficacies. Robust humoral, mucosal, and cellmediated immune responses were observed in mice immunized with the rAd5-YFV vaccine using a 2-dose vaccination regimen. The animals were fully protected from challenge with either parental $Y$. pestis CO92 or its isogenic F1 mutant in bubonic and pneumonic plague mouse models, with complete clearing of the pathogen. Furthermore, our data suggested that the T3SS needle structure protein YscF might be an important component in the rAd5-YFV vaccine formulation, especially against F1-negative $Y$. pestis CO92 challenge.

\section{RESULTS}

A 2-dose rAd5-YFV trivalent needle-free vaccination regimen provides complete protection to mice against plague

We have previously shown that a single dose administration of the rAd5-YFV vaccine $\left(8.0 \times 10^{9}\right.$ v.p.) by the i.n. route to mice provided complete protection against bubonic plague (subcutaneous, s.c., challenge), while $60 \%$ protection was achieved against pneumonic plague (i.n. challenge) ${ }^{24}$. Similar levels of protection were observed even when pre-existing immunity to Ad5 vector was induced in mice prior to vaccination ${ }^{24}$. Further, immunization of animals with the Ad5 vector alone did not influence humoral- and cell-mediated immune responses ${ }^{24}$.

To improve efficacy of the rAd5-YFV vaccine against pneumonic plague, our first attempt was to increase the i.n. immunization dose from $8.0 \times 10^{9}$ v.p. to $1.2 \times 10^{10}$ v.p., and use vaccination regimen of either 1 dose or 2 doses in mice (Fig. 1a, b). As shown in Fig. 1c, animals immunized with 2 doses of the rAd5-YFV vaccine were completely protected $(100 \%)$ compared to when animals received 1 dose of the vaccine ( $88 \%$ protection). In these experiments, animals were challenged $\sim 3$ weeks post last immunization dose (Fig. 1b). Further, while immune response analysis showed a similar percentage of $\mathrm{CD}^{+} \mathrm{IFNy}^{+} \mathrm{T}$ cells between these two groups of immunized mice (Fig. 1e), a significantly higher percentage of $\mathrm{CD}^{+} \mathrm{IFN}^{+}$T-cell population was noted when animals were immunized with 2 doses of the vaccine over the 1-dose vaccination group (Fig. 1d). These data indicated that a better T-cell immune response was elicited with a 2-dose vaccination regimen. 
a

\begin{tabular}{|c|c|c|}
\hline Groups & $\begin{array}{l}\text { Vaccine and route of delivery } \\
\text { (i.n.), number of doses }\end{array}$ & Challenge $\left(100 \mathrm{LD}_{50}\right)$ \\
\hline Control $(\mathrm{n}=5)$ & PBS, 2 doses & \multirow{2}{*}{ CO92-lux (i.n.) } \\
\hline Vaccinated $(\mathrm{n}=7)$ & rAd5-YFV or rAd5-LcrV, 2 doses & \\
\hline Control $(n=5)$ & PBS, 2 doses & \multirow{2}{*}{ CO92-lux (s.c.) } \\
\hline Vaccinated $(\mathrm{n}=7)$ & rAd5-YFV or rAd5-LcrV, 2 doses & \\
\hline Control $(n=5)$ & PBS , 2 doses & \multirow{2}{*}{$\begin{array}{l}\text { F1 mutant of CO92 } \\
\text { (i.n.) }\end{array}$} \\
\hline Vaccinated $(n=7)$ & rAd5-YFV or rAd5-LerV, 2 doses & \\
\hline
\end{tabular}

b

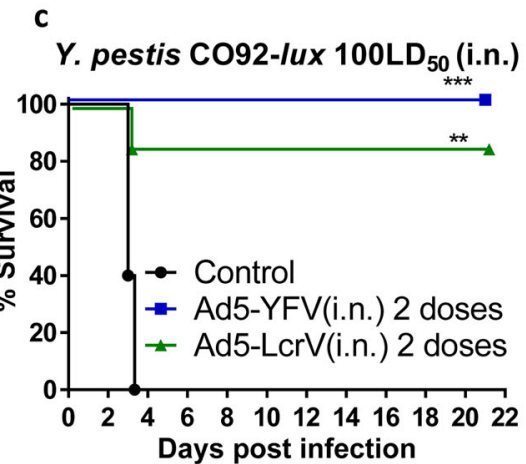

\section{d}

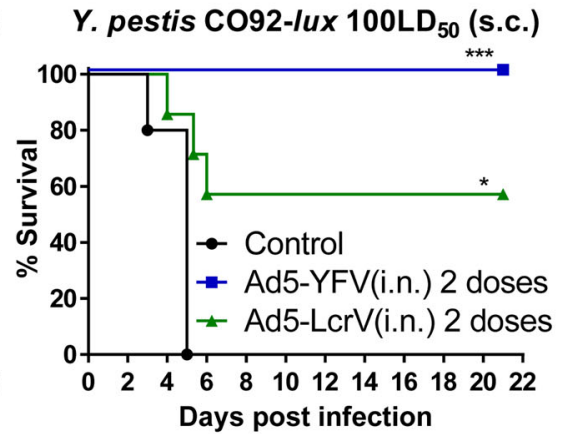

e

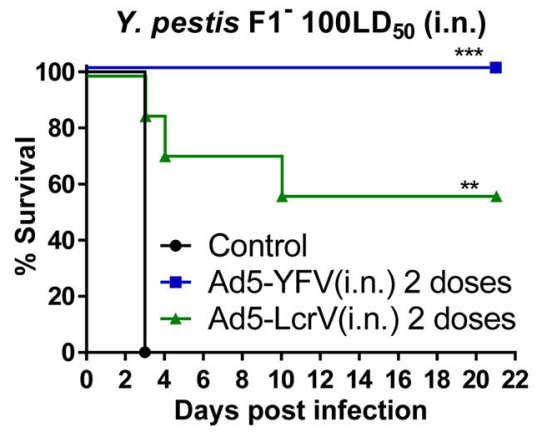

Fig. 2 Animal protection conferred by immunization of mice with rAd5-YFV or rAd5-LcrV vaccines. Mice ( $n=5-7 / g r o u p)$ were immunized (i.n.) twice 21 days apart with either $1.2 \times 10^{10}$ v.p. of rAd5-YFV or rAd5-LcrV vaccines, with mice receiving PBS served as controls (a). After 24 days post second immunization, animals were challenged with $100 \mathrm{LD}_{50}$ of $Y$. pestis CO92-lux (b) by either i.n. route (c) or s.c. route (d) or by $Y$. pestis CO92 F1-negative strain via the i.n. route (e). The percent of animal survival was then plotted. $P$ values were calculated using Kaplan-Meier analysis with log-rank (Mantel-Cox) test. Asterisks represent comparison to the control group. ${ }^{*} P<0.05,{ }^{* *} P<0.01,{ }^{* * *} P<0.001$. Two biological replicates were performed, and data plotted.

To further evaluate the 2-dose immunization strategy with the rAd5-YFV vaccine, we included the F1-minus CO92 strain in addition to the parental CO92 for challenge studies. The capsular antigen $\mathrm{F} 1$ is one of the three immunogens in the rAd5-YFV vaccine, and F1-negative strains of $Y$. pestis exist in nature that are fully virulent. Thus, with the F1-minus CO92 challenge, the protection conferred by the rAd5-YFV vaccine is only dependent on antibodies to LcrV and YscF. Therefore, for comparison, we also included the monovalent vaccine rAd5-LcrV in the study to assess the role of YscF in the rAd5-YFV vaccine when challenging immunized mice with the F1-minus CO92 strain.

As expected, both trivalent and monovalent vaccines provided protection to immunized mice albeit to varying degrees (Fig. 2). Various animal groups and the timeline for vaccination and challenge have been provided in Fig. $2 \mathrm{a}$, b. When mice were challenged with the parental $Y$. pestis CO92 strain, we observed complete protection (100\%) in the rAd5-YFV vaccine-immunized group, and an impressive $86 \%$ protection in the rAd5-LcrV vaccinated group, in a pneumonic plague mouse model (Fig. 2c). The efficacy of the rAd5-LcrV monovalent vaccine was decreased in a bubonic challenge model with only a $57 \%$ protection rate, while the $\mathrm{rAd5}$-YFV trivalent vaccine provided $100 \%$ protection (Fig. 2d). Importantly, when the immunized mice were challenged with the F1-minus $Y$. pestis CO92 strain in a pneumonic plague model, the rAd5-YFV trivalent vaccine still conferred complete protection to mice, while the rAd5-LcrV monovalent vaccine provided only $57 \%$ protection (Fig. 2e).

\section{The rAd5-YFV trivalent vaccine elicits better humoral immune response than the rAd5-LcrV monovalent vaccine}

We measured antibody titers to $F 1$ and $V$ in the sera of mice immunized with both the trivalent and monovalent vaccines using a most commonly used purified $\mathrm{rF1-V}$ fusion protein as the source of antigen. Various animal groups and the timeline for vaccination have been provided in Fig. 3a, b. A significant increase in anti-F1-V IgG antibody titers was noted after the second dose of rAd5-YFV vaccine. Importantly, the trivalent vaccine mounted significantly higher anti-F1-V IgG antibody titers compared to the rAd5-LcrV monovalent vaccine after 2 doses of the vaccination regimen (Fig. 3c).

To further profile the antibody titers, we measured IgG antibodies against the individual antigens (F1, LcrV, and YscF). We observed the same boosting effect on antibody production with significant increases in IgG titers against all three antigens after the second dose of the rAd5-YFV vaccine (Fig. 3d). As expected, no antibodies against F1 or YscF were detected in sera from mice vaccinated with the rAd5-LcrV monovalent vaccine. Further, antibody levels against LcrV were lower in mice vaccinated with 2 doses of the rAd5-LcrV monovalent vaccine than in mice that had been administered 2 doses of the rAd5-YFV trivalent vaccine (Fig. 3d). Interestingly, the antibody titer to YscF was the highest in the rAd5-YFV trivalent vaccine group of mice followed by antibody titers to F1 and then LcrV, indicating variation in immunogenicity and/or epitopes of the three antigens that were exposed in the rAd5-YFV vaccine (Fig. 3d). Some variations in the $\lg$ G titers in Fig. $3 c$, $d$ could be reflective of antigens that were used to coat the microtiter plates and/or avidity of the antibodies to F1-V or individual F1, LcrV, and YscF antigens. Further, since antibody levels to $F 1$ were higher than that of LcrV (Fig. 3d), it is expected that antibody titers to F1-V (Fig. 3c) would be similar to that of F1 titers observed in Fig. 3d.

Regarding isotypes of antibodies generated to F1-V, slight increases in both $\lg \mathrm{G} 1$ and $\lg \mathrm{g} 2 \mathrm{a}$ titers were observed after the second dose of the rAd5-YFV trivalent vaccine (Fig. 3e). Both rAd5YFV trivalent- and rAd5-LcrV monovalent-vaccines generated similar levels of IgG1 and IgG2a against F1-V (Fig. 3e) although 


\begin{tabular}{|c|c|}
\hline Groups & Vaccine, route of delivery, and number of doses \\
\hline Control (n=5) & PBS (i.n.), 2 doses \\
\hline Vaccinated (n=7) & rAd5-YFV (i.n.), 2 doses \\
\hline Vaccinated ( $\mathrm{n}=7)$ & rAd5-LcrV (i.n.), 2 doses \\
\hline
\end{tabular}

c

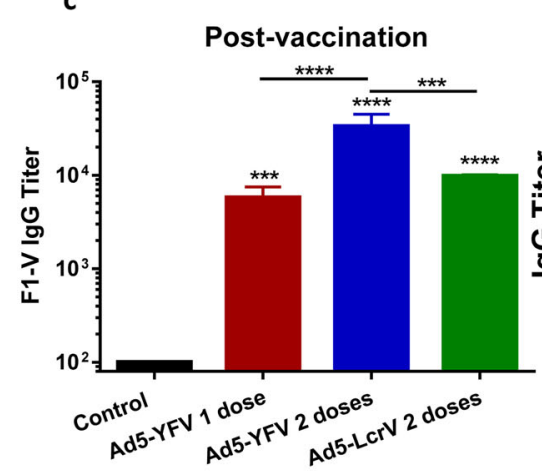

d

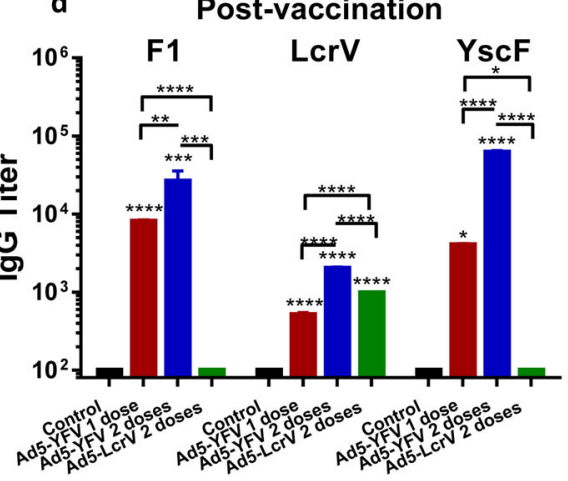

b

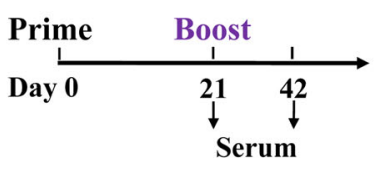

e

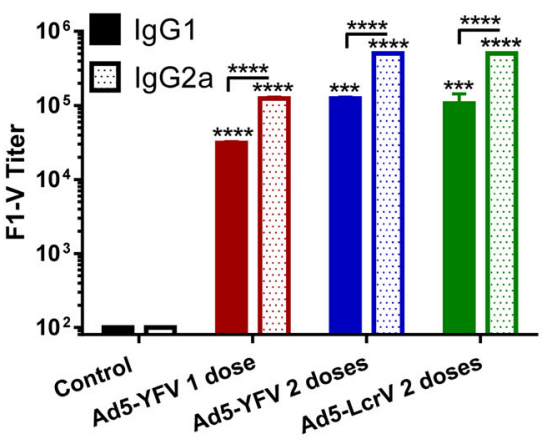

Fig. 3 Antibody responses generated in mice immunized with rAd5-YFV or rAd5-LcrV vaccines. Mice ( $n=5-7 / g r o u p)$ were immunized (i.n.) twice 21 days apart with $1.2 \times 10^{10}$ v.p. of rAd5-YFV or rAd5-LcrV vaccines, with animals that received PBS served as controls (a, b). Serum was collected on day 21 after the first dose as well as after the second dose of immunization (day 42) (b). The IgG antibody titers to rF1-V fusion protein (c) or to each individual antigen $\mathrm{rF1}$, rLcrV, and $\mathrm{rYscF}(\mathbf{d})$, as well as the IgG1 and lgG2a isotype titers to rF1-V fusion protein (e), were measured by ELISA. The geometric mean of each sample \pm standard deviations was used for data plotting. $P$ values were calculated using a one-way or two-way ANOVA with Tukey's post-hoc test to compare multiple groups or Student's $t$-test to compare two groups. Asterisks above columns represent comparison to the control group, while horizontal bars represent differences between test groups. ${ }^{*} P<0.05$, ${ }^{* *} P<0.01$, ${ }^{* * *} P<0.001,{ }^{* * * *} P<0.0001$. Two biological replicates were performed, and data plotted.

the $\mathrm{OD}_{450 \mathrm{~nm}}$ readings were higher for the rAd5-YFV trivalent vaccine than that of the rAd5-LcrV monovalent vaccine at a given dilution, without overall affecting the antibody titers. Further, significantly higher levels of $\operatorname{lgG} 2 \mathrm{a}$ compared to that of $\operatorname{lgG} 1$ indicated a stronger Th1-biased immune response that was generated by both the vaccines (Fig. 3e).

\section{The rAd5-YFV trivalent vaccine induces better cell-mediated immune response compared to that of the rAd5-LcrV monovalent vaccine}

We then compared cell-mediated immune responses generated by the trivalent versus the monovalent rAd5 vaccine. This was accomplished by measuring specific $\mathrm{T}$ - and B-cell proliferation in response to $\mathrm{rF1}-\mathrm{V}$ stimulation (ex vivo) post-immunization as well as in response to $Y$. pestis infection (in vivo) on day 3 postchallenge of mice (Fig. $4 a, b$ ). The proliferation of immune cells was evaluated by measuring bromodeoxyuridine (BrdU) incorporation in dividing cells.

As shown in Fig. 4c, we observed higher T-cell proliferation in rAd5-YFV trivalent vaccine-immunized mice than that in the rAd5LcrV monovalent vaccinated animals during both postimmunization and post-challenge. On the other hand, both rAd5YFV trivalent- and rAd5-LcrV monovalent- vaccine-immunized mice had a similar level of B-cell proliferation, although their proliferation levels were significantly higher compared to their respective controls (Fig. 4d). Our earlier studies have convincingly shown that Ad5 vector- and rAd5-YFV-vaccinated mice did not contribute to any nonspecific cellular immune responses, including T-cell proliferation $^{24}$. The cellular immune responses were only triggered after stimulating $T$ cells with $Y$. pestis specific antigens.

To further investigate the T-cell response, interferon gamma (IFNץ) production was measured by flow cytometry both during post-immunization and at 3-day post-challenge with parental $Y$. pestis $\mathrm{CO} 92$ in mice (Fig. 4b). $\mathrm{CD}^{+}{ }^{+} \mathrm{T}$ cells from the rAd5-YFV trivalent vaccine-immunized mice had a significantly higher percentage of IFN $\gamma$ producing cells compared to the respective control, as well as to the rAd5-LcrV monovalent vaccineimmunized animals (Fig. 4e). There was no difference in percentage of $\mathrm{CD}^{+} \mathrm{IFNY}^{+} \mathrm{T}$ cells between rAd5-YFV trivalent and rAd5-LcrV monovalent vaccine-immunized mice, but their percentages were increased in both the vaccinated groups as compared to their respective control groups of mice (Fig. 4f). No nonspecific IFN $\gamma$ production was noted when T cells from the Ad5 vector- and rAd5-YFV-vaccinated mice remained unpulsed, but significantly higher levels of IFN $\gamma$ was produced when T cells were pulsed with $\mathrm{rF1}-\mathrm{V}$, based on ELISpot ${ }^{24}$.

We also measured cytokine and chemokine production in the splenocyte culture supernatants (Supplementary Fig. 2A-a and b). Compared to the respective controls (Supplementary Fig. 2B-D), the overall mouse cytokines and chemokines induced by either rAd5-YFV trivalent or rAd5-LcrV monovalent vaccine immunizations were similar in terms of their trends and levels. Significantly higher levels of proinflammatory cytokines IL-1a, IL-1 $\beta$, and IL- 6 were observed in control mice during infection. However, their levels remained low in either rAd5-YFV trivalent or rAd5-LcrV monovalent vaccinated animals both during immunization and post-challenge (Supplementary Fig. 2B). Significantly higher amounts of IFNY, IL-4, and GM-CSF were observed in the rAd5YFV trivalent vaccine-immunized mice when compared to animals vaccinated with the rAd5-LcrV monovalent vaccine in response to infection (Supplementary Fig. 2C, D). On the other hand, IL-13 and GM-CSF levels were substantially elevated in the rAd5-LcrV monovalent vaccinated mice (prior to infection) as compared to animals that were immunized with the rAd5-YFV trivalent vaccine (Supplementary Fig. 2C, D). 


\begin{tabular}{|c|c|c|}
\hline $\begin{array}{c}\text { Groups } \\
(\mathbf{n}=\mathbf{1 0} \text { /group) }\end{array}$ & $\begin{array}{c}\text { Vaccine, route of } \\
\text { delivery, and } \\
\text { number of doses }\end{array}$ & $\begin{array}{c}\text { Vaccinated and } \\
\text { challenged, dose, and } \\
\text { route }\end{array}$ \\
\hline Control & PBS (i.n.), 2 doses & \multicolumn{1}{|c|}{} \\
\cline { 1 - 2 } Vaccinated & $\begin{array}{r}\text { rAd5-YFV (i.n.), 2 } \\
\text { doses }\end{array}$ & $\begin{array}{c}\text { CO92-lux }\left(100 \mathrm{LD}_{50}\right), \\
\text { i.n. }\end{array}$ \\
\cline { 1 - 2 } Vaccinated & $\begin{array}{c}\text { rAd5-LcrV } \\
\text { (i.n.), 2 doses }\end{array}$ \\
\end{tabular}

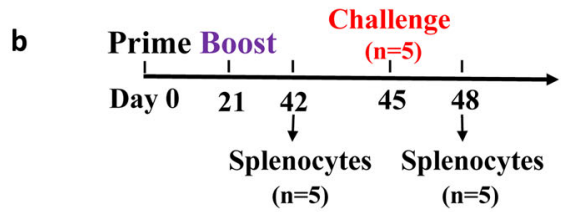

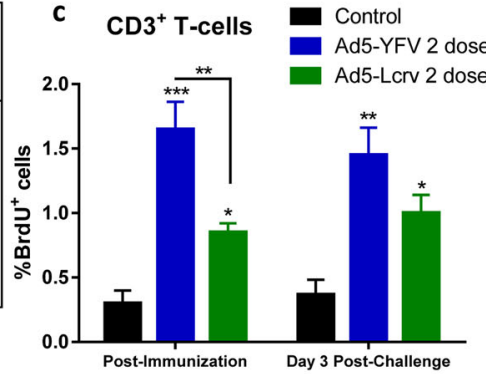

e

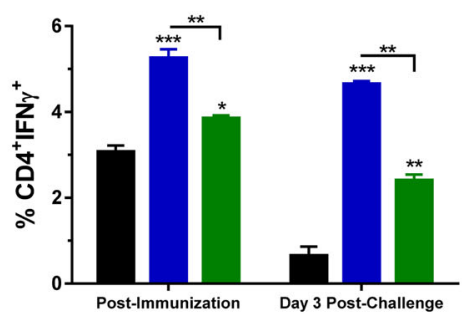

d
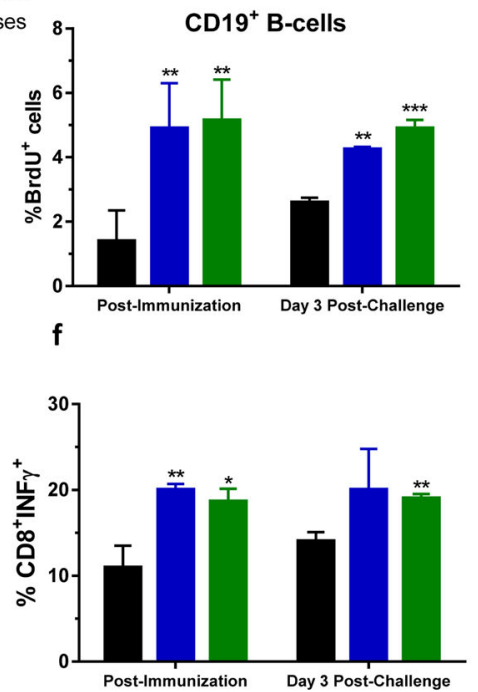

Fig. 4 Cell proliferation and IFN $p$ production in mice immunized with either rAd5-YFV or the rAd5-LcrV vaccines. Mice ( $n=10 /$ group) were immunized (i.n.) twice 21 days apart with indicated vaccines as described in panel a. Spleens were harvested from mice $(n=5)$ either 21 days after the second vaccination dose or on day 3 post i.n. challenge with $100 \mathrm{LD}_{50}$ of $Y$. pestis CO92-lux (b). For cell proliferation study after the second vaccination dose, splenocytes were stimulated with rF1-V $(100 \mu \mathrm{g} / \mathrm{ml})$ for $72 \mathrm{~h}$ at $37^{\circ} \mathrm{C}$, and then $10 \mu \mathrm{M}$ BrdU was added during the last $18 \mathrm{~h}$ of incubation with the recombinant protein. For post-challenge timepoint, mice $(n=5)$ was i.n. challenged on day 24 after immunization (day 45 of the study) with Y. pestis CO92-lux (b), and $1 \mathrm{mg} / 100 \mu \mathrm{L}$ BrdU was i.p. injected daily into mice for 3 days. On day 3 p.i., spleens were harvested after $1 \mathrm{~h}$ of last BrdU injection. The harvested splenocytes were then stained for T- and B-cell surface markers (CD3 and CD19) as well as for incorporated BrdU, and analyzed by flow cytometry. The percent of BrdU incorporation in CD3 (c) or CD19 (d) positive cells was plotted. For IFN $\gamma$ studies (e, f), splenocytes were first stimulated with PMA and lonomycin. Brefeldin A was then added to prevent secretion of cytokines. Cells were stained with T-cell surface markers CD3, CD4, and CD8 followed by intracellular IFN $\gamma$ staining. Percentage of $\mathrm{CD}^{+} \mathrm{IFN}^{+}(\mathbf{e})$ and $\mathrm{CD}^{+} \mathrm{IFN} \gamma^{+}$cells (f). $P$ values were calculated using a one-way ANOVA with Tukey post-hoc test to compare multiple groups or student t-test to compare rAd5-LcrV CD8 ${ }^{+} \mathrm{IFN} \gamma^{+} \mathrm{T}$ cells to control during post-challenge timepoint. Asterisks above columns represent comparison to the control group, while horizontal bars represent differences between test groups. ${ }^{*} P<0.05,{ }^{* *} P<0.01,{ }^{* * *} P<0.001$. Two biological replicates were performed, and data plotted.

\section{The rAd5-YFV trivalent vaccination regimen induces a sustained protective immune response}

To assess the long-term protective immune responses, the immunized mice were challenged 85 days (12 weeks) after immunization (Fig. 5a, b), and the progression of infection monitored by whole body in vivo imaging. As shown in Fig. $5 \mathrm{c}$ at day 3 post-infection (p.i.), a strong bioluminescence detected in the challenged control mice was indicative of $Y$. pestis CO92-lux (with the luciferase gene) dissemination. However, the rAd5-YFVimmunized and challenged animals did not exhibit any bioluminescence at day 3 p.i., indicating clearing of the pathogen. All control mice in both $Y$. pestis CO92-lux and its F1-minus strain challenged groups went to moribund stage on day 3 p.i., while $100 \%$ of the rAd5-YFV-immunized ones survived from the challenges (Fig. $5 \mathrm{~d}, \mathrm{e}$ ), correlating with nondetectable number of Y. pestis CO92-lux on day 3 p.i. (Fig. 5c, f). Furthermore, animal mortality was correlated with high numbers of plague bacilli present in multiple organs (lungs, liver, and spleen) of moribund control mice on day 3 p.i., while no $Y$. pestis was detected in the organs of rAd5-YFV-immunized and challenged mice neither on day 3 p.i. (Fig. 5c, based on bioluminescence) nor on day 28 p.i., based on standard bacterial plate counts (Fig. $5 f$ ).

We also observed that immunized mice maintained high IgG antibody titers to F1, LcrV, and YscF in sera on day 3 p.i. with $Y$. pestis CO92-lux (compare Fig. 3d and Fig. $5 \mathrm{~g}$ ). We also measured serum IgA levels against all three antigens on days 63 and 133 (Fig. 5b). As noted in Fig. 5h, higher IgA antibody titers were noted against $\mathrm{F} 1$ and $\mathrm{YsCF}$, with relatively lower IgA titers to LcrV, data which matched IgG antibody titers (Fig. 5g). After 28 days postchallenge (day 133), a significantly higher IgA titers to rF1-V were also detected in the bronchoalveolar lavage fluid (BALF) collected from the rAd5-YFV-immunized mice that survived the $Y$. pestis CO92-lux infection when compared to BALF collected from the uninfected control animals (Fig. 5h).

We also observed that the rAd5-YFV immunized mice (Fig. 6a, b) had significantly higher levels of proliferating T cells (Fig. $6 \mathrm{C}$ ) and $B$ cells (Fig. 6d) post-immunization as well as on day 3 postchallenge with $Y$. pestis $\mathrm{CO} 22$ as compared to their appropriate controls (Fig. 6a). Similarly, the rAd5-YFV vaccinated mice had higher percentages of $\mathrm{CD}^{+}{ }^{+} \mathrm{IFN} \gamma^{+}$T cells (Fig. 6e) and $\mathrm{CD}^{+} \mathrm{IFNY}^{+}$ T cells (Fig. 6f) compared to their respective controls (Fig. 6a) both at post-immunization and post-challenge of vaccinated mice.

\section{The rAd5-YFV trivalent vaccination regimen elicits strong cytokine and chemokine production in the long-term study}

We measured cytokine and chemokine production by splenocytes on day 63 post-immunization and day 3 post-challenge with parental $Y$. pestis CO92 strain (Fig. 7a, b). Based on our earlier studies $^{26}$, we have shown that with the live-attenuated plague vaccines, significant levels of $\mathrm{CD} 19^{+} \mathrm{CD} 38^{+}$lgG memory B cells could be detected until day 63 . However, by day 84 , the percentage of these memory $B$ cells were similar to that of the control. Similarly, percentage of $\mathrm{CD}^{+}{ }^{+} \mathrm{T}$ cells producing $\mathrm{IL}-17$ remained high until day 63 , declining to that of control by day 84 . Based on these data, we chose to examine cytokines/chemokines on day 63 post-immunization ${ }^{26}$.

In response to rAd5-YFV vaccination, the overall cytokines and chemokines in mice were either elevated or remained at compatible levels to those of control mice prior to infection (Fig. 7a, b). However, significant changes in cytokine and chemokine levels were observed during infection of immunized mice. 
a

\begin{tabular}{|c|c|c|}
\hline Groups & $\begin{array}{c}\text { Vaccine, route of } \\
\text { delivery, and number } \\
\text { of doses }\end{array}$ & $\begin{array}{l}\text { Challenge strain } \\
\left(100 \mathrm{LD}_{s,}, \mathrm{~L} \text {..n.) }\right.\end{array}$ \\
\hline $\begin{array}{c}\text { Control } \\
(n=5)\end{array}$ & PBS (i.n.), 2 doses & \multirow{2}{*}{$\operatorname{CO} 92-\ln x$} \\
\hline $\begin{array}{c}\text { Vaccinated } \\
\quad(n=8)\end{array}$ & $\begin{array}{l}\text { rAd5-YFV (i.n.), } 2 \\
\text { doses }\end{array}$ & \\
\hline $\begin{array}{l}\text { Control } \\
(\mathrm{n}=5)\end{array}$ & PBS (i.n.), 2 doses & \multirow{2}{*}{ Fl mutant of $\mathrm{CO} 92$} \\
\hline $\begin{array}{l}\text { Vaccinated } \\
\quad(n=8)\end{array}$ & $\begin{array}{c}\text { rAd5-YFV (i.n.), } 2 \\
\text { doses }\end{array}$ & \\
\hline
\end{tabular}

c

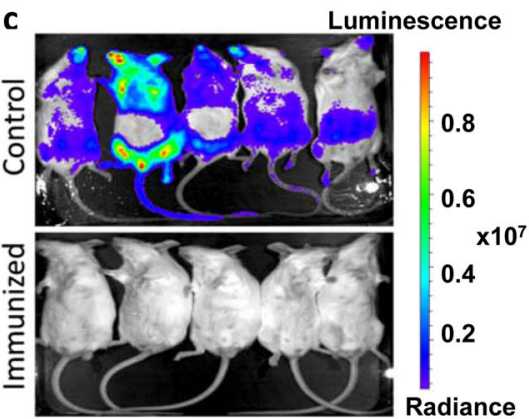

$\left(\mathrm{p} / \mathrm{sec} / \mathrm{cm}^{2} / \mathrm{sr}\right)$

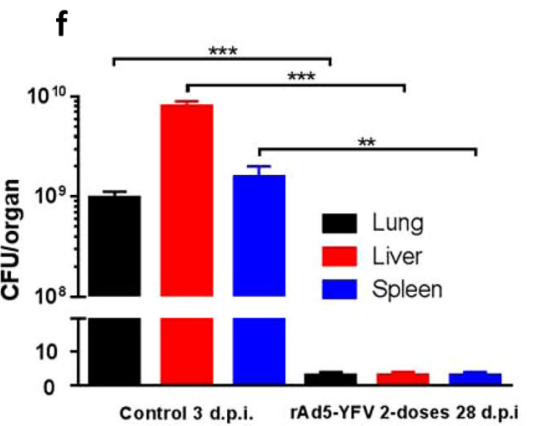

d
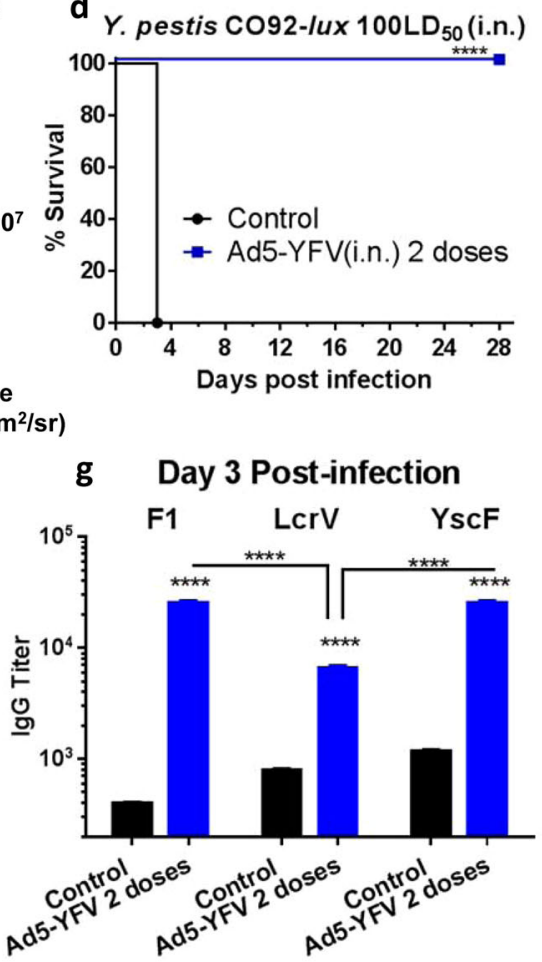

b

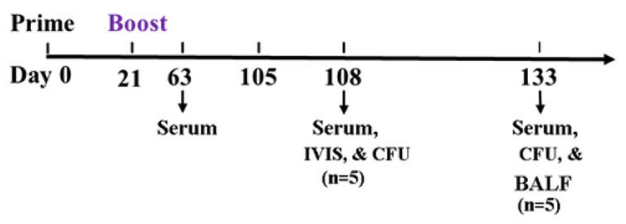

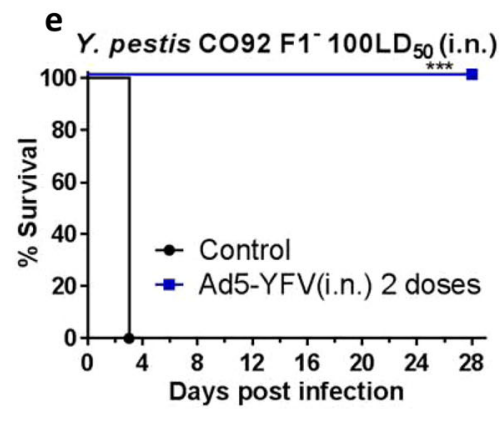

h Serum

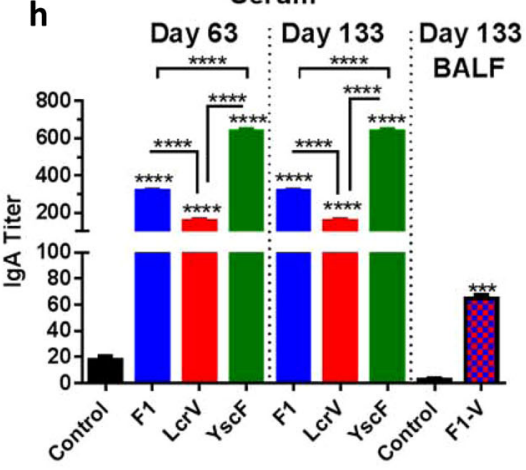

Fig. 5 Long-term protection and antibody titers in mice immunized with 2 doses of the rAd5-YFV vaccine. Mice ( $n=5-8 /$ group) were immunized (i.n.) twice 21 days apart with $1.2 \times 10^{10}$ v.p. of the rAd5-YFV vaccine, with animals receiving PBS served as controls (Fig. $5 a$ ). Eightyfour days (day 105 of study) after last vaccination, mice were i.n. challenged with $100 \mathrm{LD}_{50}\left(5 \times 10^{4} \mathrm{CFU} / 40 \mu \mathrm{L}\right)$ of $Y$. pestis CO92-lux or $Y$. pestis CO92 F1-minus strain (Fig. 5b). The progression of infection in Y. pestis CO92-lux challenged mice was monitored by IVIS, and representative images of mice with a heat map of bacterial burden from lowest (violet) to highest (red) at day 3 p.i. are shown (Fig. 5c). The percent of animal survival was plotted (d) for the Y. pestis CO92-lux challenge or (e) for its F1-minus strain challenge. The actual bacterial loads in moribund control mice at day 3 p.i., and in the rAd5-YFV immunized mice at day 28 p.i. is displayed in panel f. On day 3 p.i., serum was collected from each group, and F1, LcrV, and YscF specific IgG titers were determined by ELISA (g). On days 63, and 133 (Fig. 5b), serum was examined for F1, LcrV, and YscF specific IgA titers by ELISA (Fig. 5h). At 28 days post-challenge, BALF was collected from mice in the $Y$. pestis CO92-lux challenged cohort and F1-V specific IgA titers were determined by ELISA (Fig. $5 \mathrm{~h}$ ). $P$ values were calculated using Kaplan-Meier analysis with log-rank (Mantel-Cox) test for animal survival. One-way or two-way ANOVA with Tukey's post-hoc test was used to compare multiple groups or Student's $t$-test to compare two groups. Asterisks above columns represent comparison to the control group, while horizontal bars represent differences between test groups. ${ }^{*} P<0.05,{ }^{* *} P<0.01,{ }^{* *} P<0.001,{ }^{* * *} P<0.0001$. Two biological replicates were performed, and data plotted.

More specifically, the proinflammatory cytokines (IL-1a, IL-1 $13, \mathrm{IL}-6$, and TNF- $a$ ), as well as an anti-inflammatory cytokine IL-10, all were elevated in challenged control mice (Fig. $7 c-g$ ), but were generally unchanged in immunized mice in response to CO92 challenge (Fig. 7c-g). In contrast, cytokines related to a Th1-immune response [IL-2, IL-12(p70), and IFNY] were significantly elevated in immunized mice, while these cytokines remained almost unchanged in control mice in response to an infection (Fig. $7 \mathrm{~h}-\mathrm{j}$ ). On the other hand, the Th2immune response cytokines (IL-4, IL-5, and IL-13) were induced in vaccinated mice compared to control animals. However, they either significantly subsided (IL-4 and IL-5) or maintained at a similar level (IL-13) in immunized mice in response to an infection with CO92 (Fig. $7 \mathrm{k}-\mathrm{m})$. It is important to note that vaccinated and challenged animals still had higher levels of IL-4 and IL-13 compared to challenged control mice (Fig. 7k, m).

For the chemokines and G-CSF (Fig. 7n-r), a similar level was observed in both control and immunized mice except for GM-CSF (Fig. 7s) which was significantly higher in immunized mice than in 


\begin{tabular}{|c|c|c|}
\hline $\begin{array}{c}\text { Groups } \\
(n=10 / \text { group })\end{array}$ & $\begin{array}{l}\text { Vaccine, route of } \\
\text { delivery, and } \\
\text { number of doses }\end{array}$ & $\begin{array}{l}\text { Vaccinated and } \\
\text { challenged, } \\
\text { dose, and route }\end{array}$ \\
\hline Control & PBS (i.n.), 2 doses & \multirow{2}{*}{$\begin{array}{l}\text { CO92-lux (100 } \\
\left.\text { LD }_{50}\right) \text {, i.n. }\end{array}$} \\
\hline Vaccinated & $\begin{array}{c}\text { rAd5-YFV (i.n.), } 2 \\
\text { doses }\end{array}$ & \\
\hline
\end{tabular}

b

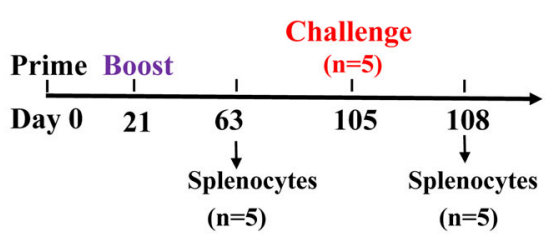

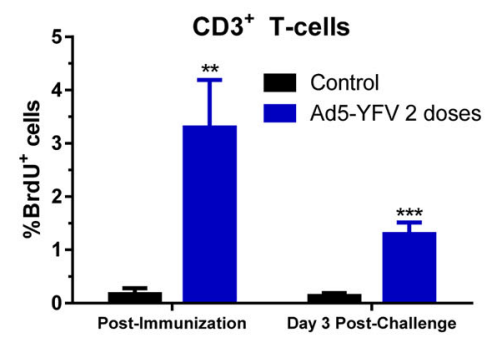

e

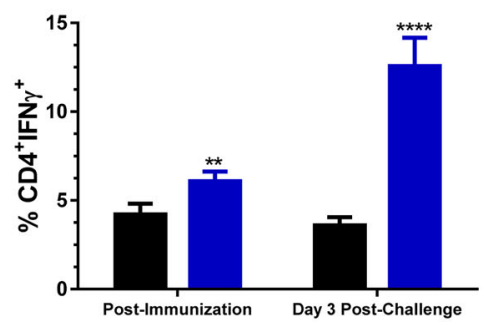

d
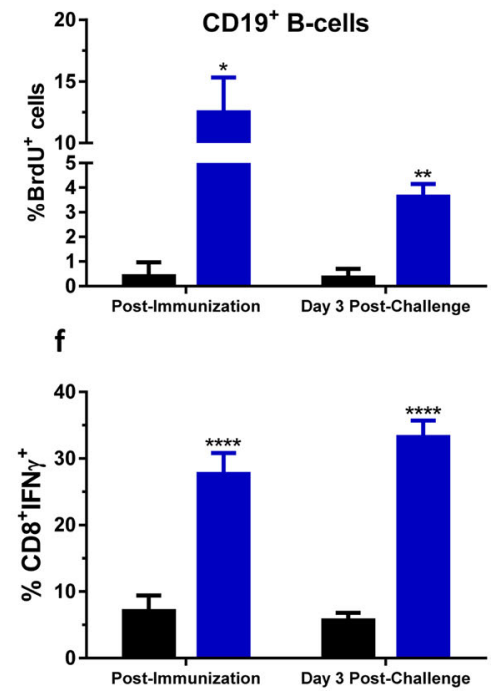

Fig. 6 Cell proliferation and IFN $\gamma$ production in mice immunized with rAd5-YFV vaccine during long-term study. Mice $(n=10 / g r o u p)$ were immunized (i.n.) twice 21 days apart with rAd5-YFV vaccine (a). Forty-two days after the second vaccination (day 63 of the study), spleens were harvested from mice $(n=5)$ and also on day 3 post-challenge with $Y$. pestis CO92-lux (b). For cell proliferation study after the second vaccination, mouse splenocytes were stimulated with $\mathrm{rF1-V}$ and treated with BrdU for the post-vaccination timepoint $(n=5)$ as described in panel b. For the post-challenge timepoint, mice $(n=5)$ was i.n. challenged on day 85 after immunization (day 105 of the study) with $Y$. pestis CO92-lux, and i.p. injected with BrdU (b). On day 3 p.i., spleens were harvested and splenocytes were then stained for T- and B-cell surface markers (CD3 and CD19) as well as for incorporated BrdU, and analyzed by flow cytometry. The percent of BrdU incorporation in CD3 (c) or CD19 (d) positive cells was plotted. For IFN $\gamma$ studies $(\mathbf{e}, \mathbf{f})$, splenocytes were stimulated with PMA, lonomycin, and Brefeldin A. Cells were then stained with T-cell surface markers CD3, CD4, and CD8 followed by intracellular IFN $\gamma$ staining. Percentages of CD4 ${ }^{+}$IFN $\gamma^{+}(\mathbf{e})$ and $\mathrm{CD} 8^{+}$IFN $\gamma^{+}$ cells (f) were shown. Student's $t$-test was used to determine statistical significance between T-cell population from control and rAd5-YFV vaccinated groups. Asterisks above columns represent comparison to the control group. ${ }^{*} P<0.05,{ }^{* *} P<0.01,{ }^{* * *} P<0.001$, ${ }^{* * * *} P<0.0001$. Two biological replicates were performed, and data plotted.

controls. However, in response to an infection, the levels of CXCL1, RANTES, and G-CSF were significantly higher in challenged control mice (Fig. $7 \mathrm{n}, \mathrm{o}, \mathrm{r}$ ). In contrast, MCP1 and CCL4 were elevated in immunized and infected mice (Fig. 7p, q), while GM-CSF (Fig. 7s) remained at high levels in both immunized as well as in immunized and challenged mice.

\section{DISCUSSION}

In the aftermath of the 2017-2018 pneumonic plague outbreak in Madagascar, the WHO released a TPP outlining the desired characteristics of a successful plague vaccine ${ }^{25}$. These characteristics included: no more than 2-dose regimen, durability of protection, needle-free administration, universal coverage against all plague-causing strains, and stability during storage ${ }^{25}$. This outbreak of 2017-2018 seemed to mirror the third pandemic, which first established plague in Madagascar ${ }^{7}$, and served as a reminder to proactively react to prevent future plague outbreaks ${ }^{7}$.

Considering number of plague cases and deaths reported to WHO from 33 countries, including the US between 2013-2018 (a case-fatality rate of $17.5 \%$ ), necessitates the development of new generation plague vaccines. Further, the recent finding that amoebae can serve as reservoirs for the plague bacterium is alarming ${ }^{7}$. Finally, the natural cellular immune response to pneumonic plague is not well understood, and to date, no phase III clinical trials have been conducted on any of the plague vaccines. Therefore, our study is timely to test a new generation plague vaccine in mice, and study in depth the immune responses.

As alluded to in our earlier paper $^{24}$, we preferred to use Ad5 vector for developing plague vaccine because of its (i) wellcharacterized viral genome and the capability of integrating multiple genes; (ii) the use of Ad5 vector for gene therapeutic applications in humans; (iii) broad tropism infecting a variety of dividing and nondividing cells; (iv) ability of adenoviruses to effectively present transgene products to antigen presenting cells in vivo to promote rapid and robust humoral and cellular immune responses; (v) preferential induction of Th1-type immune responses, alleviating Th2-mediated eosinophil-related immunopathology; (vi) ability of adenoviruses to replicate to high titers in tissue culture cells; (vii) low biocontainment requirements for producing adenoviral vaccines, (viii) can be applied systemically as well as through mucosal surfaces, and adenoviruses are relative thermostable to facilitate their clinical use; (ix) inexpensive production, $(x)$ no need for an adjuvant, and the vaccine can be delivered as aerosol mist stimulating potent and long-lasting humoral- and cell-mediated immune responses, and (xi) plug and play platform to develop multivalent/multicomponent vaccines.

Ad5 vector was also used in evaluating the efficacy of monovalent Ad5-F1 and Ad5-LcrV vaccines in a pneumonic plague mouse model using only the parental $Y$. pestis CO92 strain; however, the vaccines were administered by the i. $\mathrm{m}$. route ${ }^{27}$. Further, we noted that while 1 dose of the rAd5-LcrV monovalent vaccine $\left(8 \times 10^{9}\right.$ v.p.) provided only $20 \%$ protection to mice in a pneumonic plague mouse model when administered by the i.n. route, no protection was observed when delivered by the i. $\mathrm{m}$. route at $90 \mathrm{LD}_{50}$ of $\mathrm{CO} 92$, in spite of high antibody titers ${ }^{24}$. Our studies also showed that i.n. administration of the vaccine bypasses pre-existing antibodies to Ad5 vector to provide protection to animals ${ }^{24}$. Although $\mathrm{Ad} 5$ is the most prevalent serotype to which humans are exposed to, it induces minimal innate proinflammatory cytokine responses compared to other adenoviral serotypes used as vaccine vectors ${ }^{28}$. 

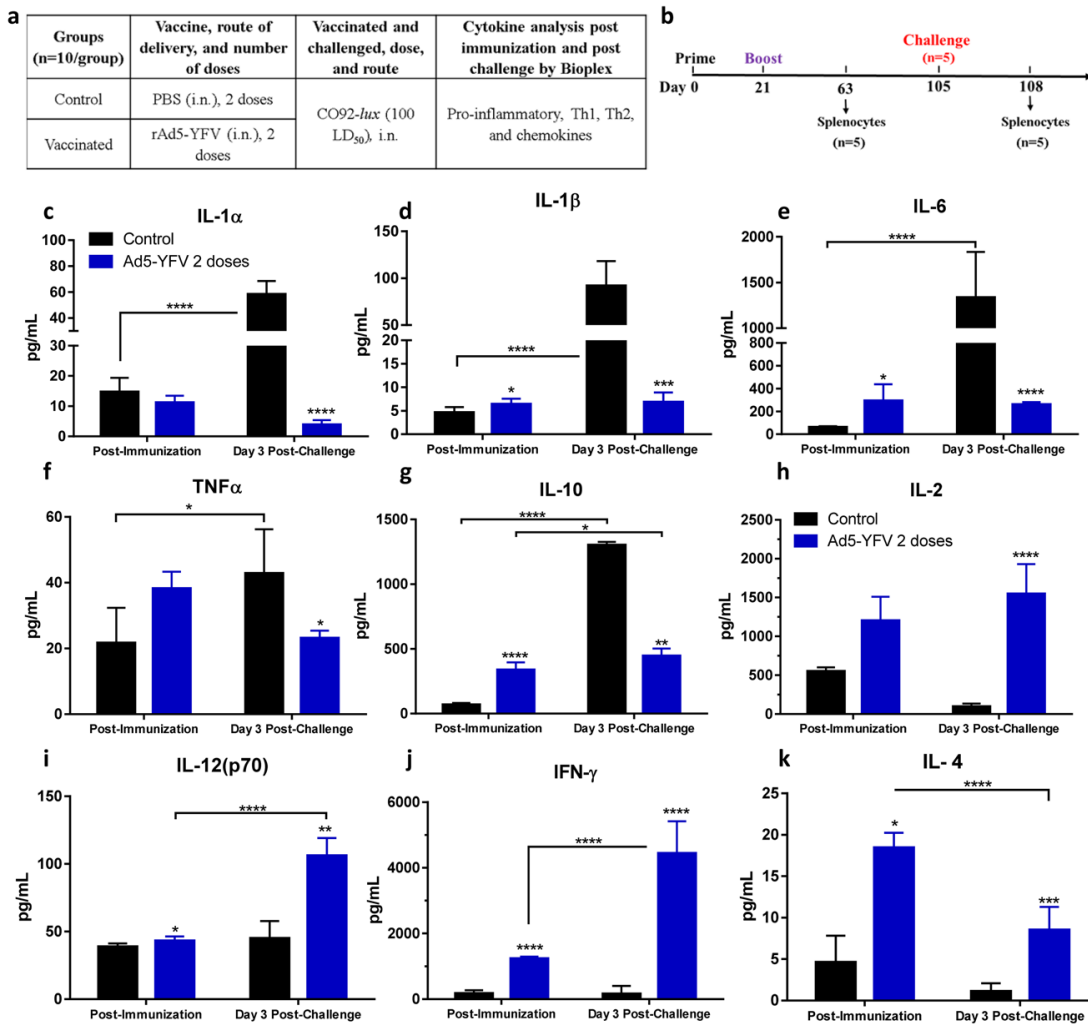

h

IL-2
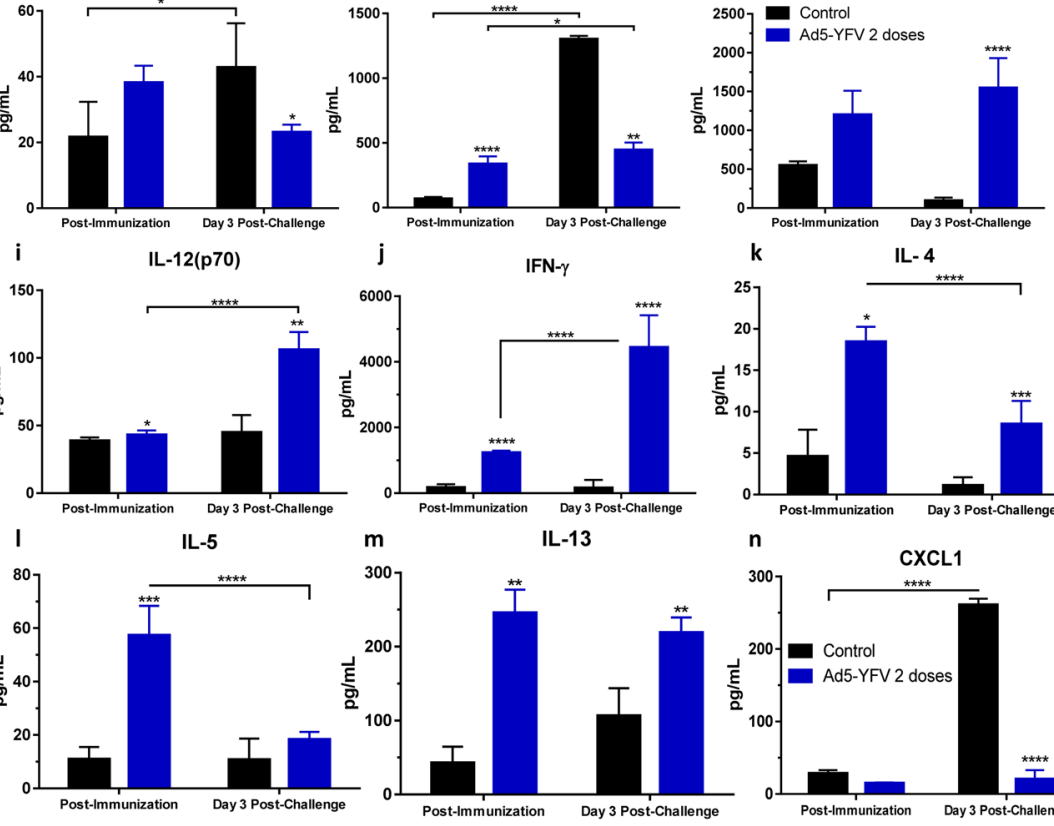

$\mathrm{F}-\gamma$

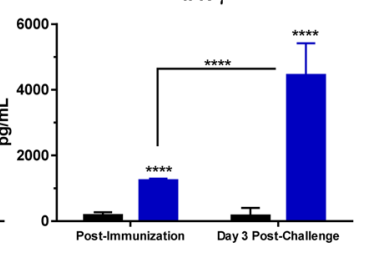

k IL- 4
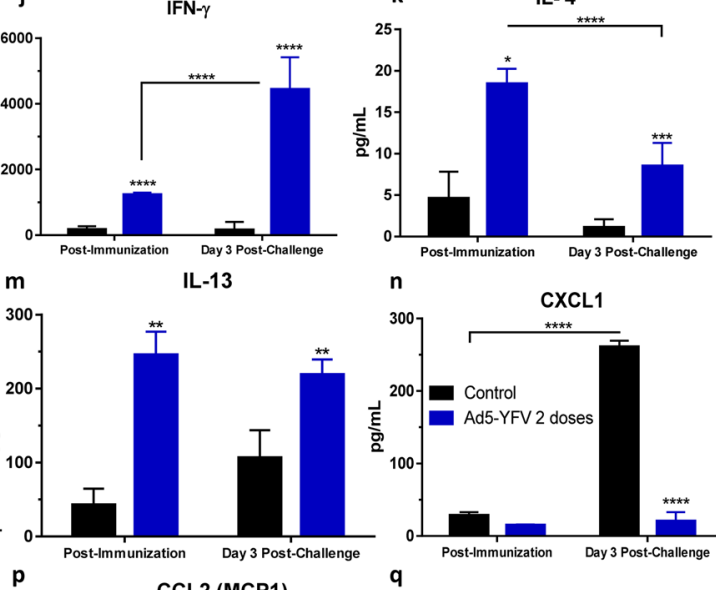

$$
\text { Post-Immunization Day } 3 \text { Post-Challenge }
$$

n
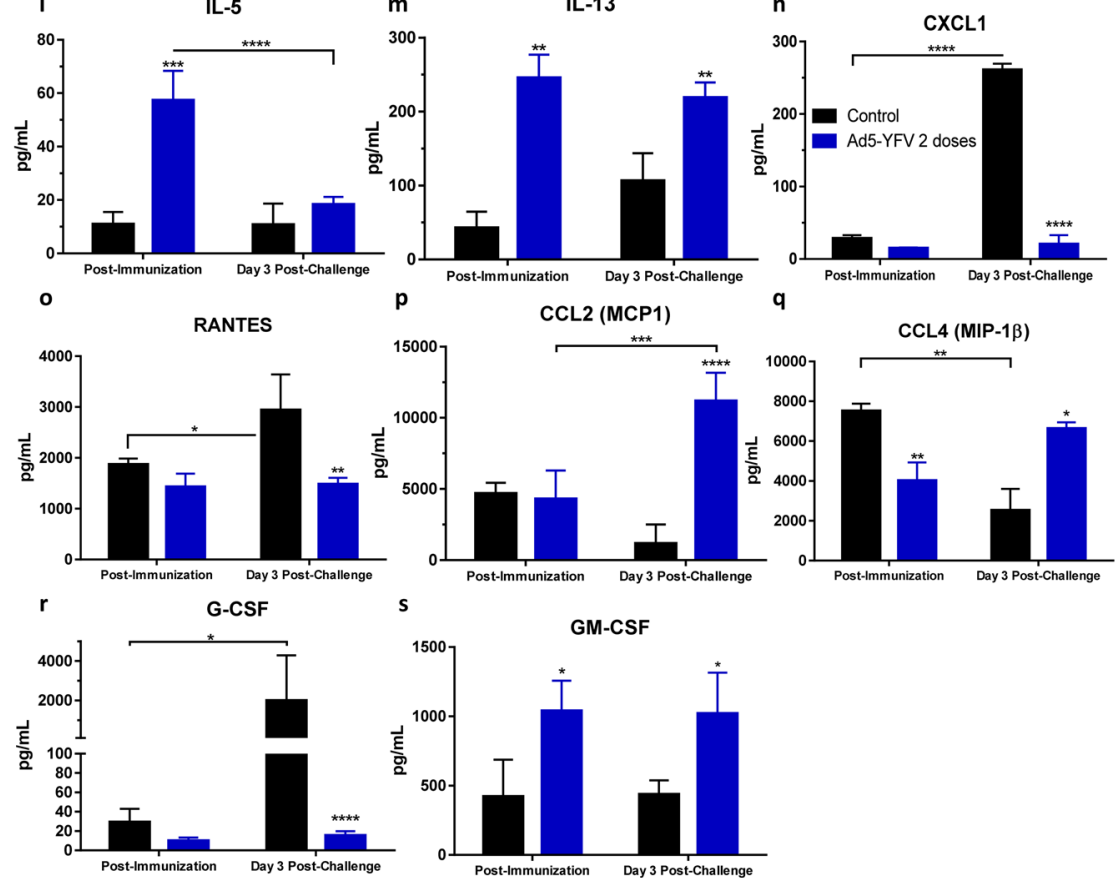

Fig. 7 Splenocyte cytokine and chemokine profiles from the rAd5-YFV vaccine-immunized mice during a long-term study. Mice ( $n=10$ / group) were immunized (i.n.) twice 21 days apart with $1.2 \times 10^{10}$ v.p. of rAd5-YFV vaccine or rAd5-LcrV vaccine, with animals receiving PBS served as controls (Fig. 7a). Forty-two days after the second vaccination dose or on day 3 post-challenge, spleens were harvested from mice ( $n=5 /$ group/timepoint) and stimulated with purified $\mathrm{rF1-V}(100 \mu \mathrm{g} / \mathrm{mL})$ for 3 days (b). The cytokines in the culture supernatants were analyzed by using Bioplex-23 assay and expressed as the arithmetic means \pm standard deviations. The proinflammatory and antiproinflammatory cytokines were listed in panel Fig. 7c-g, while the Th1 and Th2 cytokines were displayed in panels $\mathbf{h}-\mathbf{m}$, and the chemokines as well as colony stimulating factors are presented in panels $\mathbf{n}-\mathbf{s}$. $P$ values were calculated using a two-way ANOVA with Tukey post-hoc test to compare multiple timepoints or student $t$-test to compare two groups within the same timepoint. Asterisks above columns represent comparison to the control group, while horizontal bars represent differences between test groups. ${ }^{*} P<0.05,{ }^{* *} P<0.01,{ }^{* * *} P<0.001,{ }^{* * * *} P<$ 0.0001. Two biological replicates were performed, and data plotted. 
Our 2-dose rAd5-YFV intranasal vaccination strategy essentially fulfills WHO's TPP. The currently developed plague vaccines which underwent phase I and II clinical trials are based on F1 and V proteins mixed with alum ${ }^{29}$ or flagellin ${ }^{13}$ adjuvants. These vaccines induce a strong humoral response against both F1 and $V$ antigens and have been shown to be efficacious in mouse and some non-human primate models of pneumonic plague. For example, consistent protection was observed in cynomolgus macaques; however, in African green monkeys, inconsistency in protection $(0-75 \%)$ was noted in multiple studies ${ }^{8,30,31}$. The precise mechanism(s) for the lack of consistent protection has not been determined; however, humoral responses generated were similar for both non-human primate (NHP) models. It has been hypothesized that cell-mediated immune responses might be needed for complete protection in African green monkeys ${ }^{8}$, which are not adequately developed by the F1-V-based subunit vaccines.

While $\mathrm{F} 1$ and $\mathrm{V}$ are important antigens for plague vaccine development, there are some concerns that highlight the importance of including other plague immunogens to a potential universal plague vaccine. F1 capsule has been shown to be important for $Y$. pestis to resist phagocytosis during infection ${ }^{32}$. Strains of $Y$. pestis that lack F1, are still fully virulent in both pneumonic and bubonic models of disease albeit with a longer disease course seen during bubonic infection ${ }^{19}$. Bio-sampling studies have estimated that naturally occurring F1-negative $Y$. pestis strains could be as prevalent as $10-16 \%{ }^{18}$.

Likewise, LCrV is a polymorphic protein which contains hypervariable regions within the $\mathrm{COOH}$-terminal half where protective residues have been identified ${ }^{20}$. Studies have shown that vaccination with LcrV from different clades than the LcrV present in the challenge strain do not offer cross-protection during $Y$. pseudotuberculosis infection ${ }^{20}$. This scenario presents a situation where a potential outbreak strain of $Y$. pestis could avoid protection offered by a F1-V-based subunit vaccine.

In some earlier studies ${ }^{30}$, the efficacy of F1-V-based subunit vaccines against challenge with F1-negative $Y$. pestis strain at 11 weeks post-immunization, were reported. Likewise, the efficacy of a mutated $Y$. pseudotuberculosis strain expressing the gene encoding Caf 1 when administered orally in mice against evoking bubonic and pneumonic plague was published ${ }^{33}$. In this study, we have evaluated efficacy of the rAd5-YFV vaccine (administered by the i.n. route) for the duration of 15 weeks of immunization, and also after challenge with F1-negative $Y$. pestis strain in a pneumonic plague model.

Our rAd5-YFV vaccine potentially could alleviate the issue of infection with either F1-negative $Y$. pestis strains or those harboring LcrV variants by the inclusion of a third antigen YscF, another component of the $\mathrm{T}_{3 \mathrm{SS}}{ }^{34}$. During parental $Y$. pestis CO92 pneumonic challenge, we observed slightly decreased protection (86\%) by the monovalent rAd5-LcrV vaccine compared to $100 \%$ protection provided by the rAd5-YFV trivalent vaccine (Fig. 2c). The decrease in protection was even more pronounced $(57 \%$ versus $100 \%$ ) in a bubonic plague model (Fig. 2d). Furthermore, during F1-negative $Y$. pestis intranasal challenge, monovalent rAd5-LcrV vaccination only provided $57 \%$ protection, while the trivalent rAd5-YFV vaccination still conferred $100 \%$ protection (Fig. 2e). The higher level of protection conferred by rAd5-YFV immunization, especially against the $\mathrm{F} 1$-negative $Y$. pestis, could be contributed to the additional antigen $\mathrm{YscF}$, as the $\mathrm{F} 1$ component in both vaccine (rAd5-LcrV and rAd5-YFV)-immunized mice would not have a role. However, we draw this conclusion with caution as LcrV epitopes might possibly be differentially exposed in rAd5-LcrV versus rAd5-YFV vaccines, which could afford better protection against infection with the latter vaccine. Such studies would be elaborated in the future.

In retrospect, we would prefer to have generated rAd5-FV vaccine as well. However, since we generated $\mathrm{F}^{-}$mutant of $Y$. pestis $\mathrm{CO}_{2} 2^{19}$, we did not feel the necessity to develop rAd5-FV vaccine. We reasoned that infecting rAd5-LcrV-immunized animals with $\mathrm{F}^{-}{ }^{-}$mutant of $Y$. pestis $\mathrm{CO} 2$ would provide the needed information for this study. We selected YscF as studies have shown that vaccination of mice with this T3SS needle structure protein provided protection to mice against subcutaneous injection with the encapsulated $Y$. pestis $\mathrm{CO}^{3} 2^{35}$, and against an intravenously injected pigmentation locus-negative $Y$. pestis KIM strain ${ }^{34}$. In addition, we have also shown that serum from animals immunized with $Y$. pestis $\mathrm{CO} 22$ reacted strongly with $\mathrm{YscF}$ based on our proteomics studies (unpublished data). Therefore, we hypothesized that the protective antigen YscF could be used in combination with $\mathrm{F} 1$ and LcrV to formulate a more effective new generation trivalent rAd5-YFV vaccine.

The rAd5-YFV vaccine elicited stronger immune responses in mice compared to that of rAd5-LcrV vaccine. First, a much higher antibody titers were observed in mice immunized with the rAd5YFV vaccine, and the higher antibodies were not only to the rF1-V fusion protein but also to each individual antigen ( $\mathrm{rF1}, \mathrm{rLCrV}$, and rYscF) (Fig. 3c, d). We noted relatively higher IgG1 and IgG2a titers with both rAd5-YFV and rAd5-LcrV vaccines compared to IgG titers (Fig. 3). Biologically, it should not be the case; however, it is not uncommon and could be reflective of binding affinity and specificity of the secondary antibodies that were used. Indeed, higher levels of IgG subclasses over IgG titers have been reported in other studies as well ${ }^{34,36-38}$. Overall, both the trivalent and monovalent vaccines generated a stronger Th1 response based on $\operatorname{lgG} 2 \mathrm{a}$ antibody titers (ratio of $\operatorname{lgG} 2 \mathrm{a} / \operatorname{lgG} 1>1{ }^{39}$. $\lg \mathrm{G} 1$ and $\lg \mathrm{g} 2 \mathrm{a}$ have a differential role in animal protection against various infections ${ }^{40,41}$.

We observed significantly higher levels of T-cell proliferation in rAd5-YFV vaccine-immunized mice than those in rAd5-LcrV vaccinated animals when splenocytes were stimulated ex vivo with rF1-V antigen and labeled with BrdU (Fig. 4c). A similar trend was also seen when BrdU was injected during challenge of immunized mice and proliferation measured $72 \mathrm{~h}$ post-infection in vivo (Fig. 4c). Likewise, we also observed a significantly increased population of $\mathrm{CD}^{+} \mathrm{IFN}^{+} \mathrm{T}$ cells both postimmunization as well as post-challenge when vaccination occurred with rAd5-YFV over rAd5-LcrV vaccine (Fig. 4e). Furthermore, substantially higher amounts of IFNY and IL-4 were produced in the splenocyte supernatants of rAd5-YFV vaccineimmunized mice than those from rAd5-LcrV vaccinated animals in response to an infection (Supplementary Fig. 2C). These data further highlighted the advantage of rAd5-YFV over rAd5-LcrV as a plague vaccine candidate.

It has been reported that F1-V-based subunit vaccines adjuvanted with alum bias the immune response towards Th2 based on antibody isotyping ${ }^{31,42}$. In contrast, both rAd5-LcrV and rAd5-YFV vaccination generated strong Th1 and Th2-immune responses and were more biased towards Th1 based on the $\operatorname{lgG} 2 \mathrm{a} / \operatorname{lgG} 1$ antibody ratio, as well as the cytokine profile with overall higher magnitude of Th1 cytokines over Th2 cytokines (Fig. $3 e$ and Supplementary Fig. 2C). A Th1-biased response is usually correlated with robust cell-mediated immunity that might be needed for complete protection in African green monkeys against pneumonic plague ${ }^{43}$.

A single dose of the rAd5-YFV vaccine provided impressive but incomplete protection (88\%) in mice after challenge with parental CO92 (Fig. 1c). We noted a significant increase in anti-F1-V IgG antibody titers after the second dose of rAd5-YFV vaccine administration (Fig. 3). This boosting effect was also observed in the cell-mediated immune response, as increased levels of antigen specific $\mathrm{CD}^{+}{ }^{+} \mathrm{IFNY}^{+}$T cells were observed in mice immunized with 2 doses of the rAd5-YFV vaccine compared to a single vaccine dose (Fig. 1d). While these data suggested that 2 doses of vaccine were needed to confer complete protection, 1 dose of the rAd5-YFV vaccine could be given during an outbreak emergency 
response scenario where there is not enough time to complete a full vaccination regimen as outlined in the TPP developed by the $\mathrm{WHO}^{25}$.

The TPP also stipulated that a viable vaccine should confer protection for at least 2 years in a reactive vaccination scenario and at least 5-10 years for an ideal preventative use scenario ${ }^{25}$. We detected high levels of antigen specific antibody titers in the rAd5-YFV vaccine-immunized mice on day 3 post-challenge, which was 88 days ( $\sim 13$ weeks) after last immunization (challenge day 108). These data suggested that 2 doses of the rAd5-YFV vaccine induced a sustained immune response in mice (Fig. $5 g$, h). It is difficult to correlate duration of protection in mouse models to length of protection that may be generated in humans due to a nonlinear relationship between life stages in mice and humans ${ }^{44}$. However, we did not observe a downward trend in antibody titers noted in other vaccine studies at similar timepoints when using 1or 2 doses of the $F 1+V$ based subunit vaccines ${ }^{45,46}$.

We also observed significant levels of F1, LcrV, and YscF specific $\lg \mathrm{A}$ antibodies in the serum of vaccinated mice that survived 4 weeks of challenge compared to the control (Fig. 5h). While we did not measure IgA antibodies in the lungs of vaccinated mice prior to challenge, we noted higher levels of F1-V specific IgA in the BALF after 4 weeks of challenge (Fig. 5h). Antigen specific IgA antibodies, especially present in the airway, are important against respiratory infections, and immunization of animals via intranasal route is believed to facilitate the stimulation of mucosa immunity.

However, it is important to note that the role of $\lg \mathrm{A}$ during pneumonic plague is not clear. In a study by Singh et al., IgA seemed dispensable in protecting mice against pneumonic plague $^{36}$. In these studies, YopE-LcrV was produced from an attenuated strain of $Y$. pseudotuberculosis, and animals were immunized by the oral route. Our future studies will fully explore the role of $\lg A$ in protection using rAd5-YFV vaccine against pneumonic plague.

In addition to the induction of high levels of antibodies during the long-term study with the 2-dose vaccination regimen, we also observed significant $\mathrm{T}$ - and B-cell proliferation along with higher percentages of $\mathrm{CD}^{+}{ }^{+} \mathrm{IFN}^{+}$and $\mathrm{CD}^{+} \mathrm{IFNy}^{+}$cells in mice both after immunization and post-challenge (Fig. 6). These data confirmed that both humoral and cell-mediated immune responses generated were robust and long-lived, and these data were further supported by the splenocyte cytokine and chemokine profiles (Fig. 7) and the rapid clearance of the invaded pathogen (Fig. 5c, f).

In control animals, we noted a significant increase in proinflammatory cytokines (IL-1a, IL-1 $\beta, \mathrm{IL}-6$, and TNF- $\mathrm{a}$ ), and chemokines (RANTES/CCL5 and CXCL1) coupled with the surge of G-CSF in response to infection (Fig. 7). This proinflammatory cytokine storm is a typical result of host neutrophil-macrophage interaction in recognition of invading pathogens ${ }^{47}$ and corresponds to the characteristic highly proinflammatory state of pneumonic plague (48-72 h p.i.), which leads to animal death if unresolved $^{48,49}$.

The overwhelming proinflammatory cytokine storm is also the main cause of death for current COVID-19 patients, and the antiinflammatory mediators, especially anti-RANTES, have been preferred for therapeutic intervention ${ }^{43}$. Importantly, this proinflammatory cytokine storm was prevented in rAd5-YFV vaccineimmunized mice after $Y$. pestis challenge, as all aforementioned cytokines and chemokines were largely unchanged when compared to their levels before infection (Fig. 7). The excessive level of IL-10 in infected control mice (Fig. 7) overall suppressed the immune response with a much-reduced level of Th1 and Th2 cytokines (Fig. 7). In contrast, the level of IL-10 remained low in immunized and challenged animals compared to that in control and infected mice (Fig. 7) with concomitant higher levels of Th1 and Th2 cytokines post-challenge (Fig. 7).
CXCL1 is an important chemoattractant for neutrophils. High levels of neutrophils are observed in uncontrolled plague infection, which correlate with the inflammatory cytokine storm. The latter results in immune-mediated damage to the lungs, contributing to the pathology seen during pneumonic plague ${ }^{50}$. On the other hand, we noted increased levels of monocyte/ macrophage associated chemokines CCL2/MCP1 and CCL4/MIP1 $\beta$ in rAd5-YFV vaccine-immunized mice that were not seen in controls in response to CO92 infection (Fig. 7). Influx of activated monocytes/macrophages to the infection site is important for controlling infection, clearing dead cells, and reconstituting damaged tissue structures. Beyond chemotaxis, both CCL2 and CCL4 are also involved in modulating host immune response. It has been reported that CCL2 influences myeloid cell behavior, which leads to enhance host defense, cellular cleanup, allergic responses, as well as prime monocytes and macrophages in response to subsequent infections ${ }^{51}$.

Likewise, CCL4 plays a central role in the normal initiation of Tcell and humoral responses by recruiting $\mathrm{CD}_{4}^{+} \mathrm{CD} 25^{+} \mathrm{T}$-cell population ${ }^{52}$. In addition, we observed higher level of GM-CSF in rAd5-YFV vaccine-immunized mice than in controls (Fig. 7). Unlike G-CSF, which was increased in control mice in response to $Y$. pestis challenge to prolong neutrophil survival (Fig. 7), GM-CSF is a growth and differentiation factor for both granulocyte and macrophage populations ${ }^{53}$. Interestingly, GM-CSF has been reported to be involved indirectly in the induction of immunological tolerance and anti-inflammatory responses. More specifically, GM-CSF has been shown to facilitate T-cell-mediated tolerance by inducing "tolerogenic" dendritic cells $(D C s)^{53,54}$. Therefore, the cytokines and chemokines elicited in rAd5-YFV vaccine-immunized mice obviously orchestrated a strong but controlled immune response to combat $Y$. pestis infection, while avoiding tissue damage, which correlated with the clearance of $Y$. pestis with no signs of disease, such as ruffled fur, lethargy, hunched posture, or lack of grooming. Our previous studies have shown that rAd5-YFV vaccinated NHPs did not show any histopathological lesions in any of the examined organs ${ }^{24}$.

In conclusion, rAd5-YFV vaccine with a 2-dose regimen induced humoral, mucosal, and cell-mediated immune responses that protected mice against pneumonic plague caused by parental $Y$. pestis $\mathrm{CO} 2$ and its F1-minus mutant. In the future, we will continue to investigate the importance of $\lg A$ antibodies and their role in protection from pneumonic plague as well as the potential for the rAd5-YFV vaccine to protect against $Y$. pestis strains with noncross-reactive LcrV variants. In addition, we will perform a more comprehensive comparison between rAd5-YFV and rAd5LcrV vaccines (e.g., comparative expression of the IcrV gene, kinetics of the immune responses generated), and the individual contribution of $\mathrm{YscF}$ and $\mathrm{F} 1$ against protection.

\section{METHODS}

\section{Bacterial strains}

Parental Y. pestis strain CO92 (also designated as CO92) is a fully virulent human pneumonic plague isolate acquired through BEI Resources (Manassas, VA). Y. pestis CO92-lux is a bioluminescent strain (with a luciferase reporter gene) created in the laboratory ${ }^{55}$. Y pestis CO92 F1 isogenic mutant was generated in our laboratory and demonstrated full virulence in pneumonic/bubonic plague mouse models ${ }^{19}$. All studies were performed in a Tier-1 select agent laboratory at UTMB within the Galveston National Laboratory (GNL), Galveston, TX.

\section{Animal studies}

Six- to eight-week-old female Swiss-Webster mice were used in all studies. The experiments were performed in the animal biosafety level 3 (ABSL-3) facilities under an approved Institutional Animal Care and Use Committee protocol, UTMB, Galveston, Texas. Our earlier studies (unpublished) have 
shown that the gender, mouse species, as well as the age of mice did not affect the results.

\section{Immunization and antibody responses}

Mice were immunized by the i.n. route with either one or two doses of the rAd5-YFV or rAd5-LcrV vaccines $\left(1.2 \times 10^{10}\right.$ v.p./dose in a $20 \mu \mathrm{l}$ volume per nostril, followed by a phosphate-buffered saline [PBS] wash, $10 \mu$ l volume). The two vaccine doses were delivered 21 days apart. Blood was collected via the retro-orbital route at the designated timepoints. The collected sera were filtered by using $0.1 \mu \mathrm{m}$ filter cartridges (MilliporeSigma Life Science Center, Burlington, MA) and the sterility examined before subsequent procedures.

Antibody titers were determined by using indirect Enzyme-linked immunosorbent assay (ELISA). Briefly, ELISA plates were coated with $1 \mathrm{ng} / \mu \mathrm{L}$ of $\mathrm{rF1}-\mathrm{V}$ (BEI Resources) or individual antigens $\mathrm{rF1}, \mathrm{LLcrV}$, or $\mathrm{rYscF}$ and allowed to adhere to the plates overnight at $4{ }^{\circ} \mathrm{C}^{24}$. Antigen coated plates were then blocked with $1 \%$ powered milk-PBS solution for $1 \mathrm{~h}$ at room temperature. Two-fold serial dilutions of sera were added to the plates and incubated for $1 \mathrm{~h}$ at room temperature. After 3 times washes with a $0.05 \%$ Tween 20 in PBS solution, horseradish peroxidase (HRP)conjugated goat anti-mouse $\lg$, $\lg$ G1, IgG2a, or $\lg A$ antibodies (Southern Biotech, Birmingham, AL) (1:8000 dilution) were then applied for $1 \mathrm{~h}$ at room temperature. After three times washes, the reaction in the plates was developed using 3,3',5,5'-Tetramethylbenzidine (TMB) solution (Sigma-Aldrich, St. Louis, MO) $(100 \mu \mathrm{L} /$ well) for $5-15 \mathrm{~min}$ at room temperature. The reaction was stopped using $2 \mathrm{~N} \mathrm{H}_{2} \mathrm{SO}_{4}(50 \mu \mathrm{L} /$ well). Color development was read on a Versamax tunable plate reader (Molecular Devices San Jose, CA) at $450 \mathrm{~nm}$. Total lgG, its lgG1, and lgG2a isotype antibody titers, as well as $\lg A$, were measured at least in 3 replicates.

\section{Animal challenge}

For the short-term studies, animals were challenged 24 days after the last dose of the vaccine administration (day 45 of the study). For the long-term study, animals were challenged 85 days after the last dose of the vaccine delivery (day 105 of the study). Mice were anesthetized with isoflurane (to inhale) and challenged with $100 \mathrm{LD}_{50}\left(1 \mathrm{LD}_{50}\right.$ corresponds to 50 or 500 colony forming units [CFU] for s.c. or i.n. challenge, respectively, to induce bubonic or pneumonic plague) by either parental $Y$. pestis CO92-lux or its F1-minus mutant ${ }^{19}$. Animals were observed for morbidity and mortality for 21-28 days. The progression of infection with $Y$. pestis CO92-lux was monitored by using an in vivo imaging system (IVIS) 200 bioluminescent and fluorescent whole body imaging system (Caliper Corp., Alameda, CA, USA) as previously described ${ }^{55}$. At designated timepoints (e.g., day 3 p.i. or the day of experiment termination), lungs, liver and spleen were harvested from animals to assess bacterial burden as we previously described ${ }^{56}$.

\section{Cell proliferation and cytokine production}

To measure T- and B- cell proliferation, bromodeoxyuridine (BrdU), a thymidine analog, incorporation method was used. Briefly, spleens were aseptically removed from five animals of each group on either day 21 or day 42 after the last immunization dose, or day 3 p.i. with $Y$. pestis-lux C092. Spleens were homogenized and passed through a $70 \mu \mathrm{m}$ cell strainer to obtain single cell suspension in RPMI 1640 cell culture medium. Spleens from control mice served as a negative control. Splenocytes were then seeded into 24 -well tissue culture plates at a density of $2.0 \times 10^{6}$ cells/ well and 4 wells/mouse/plate. For post-vaccination cell proliferation studies (ex vivo), the isolated splenocytes were first stimulated with rF1$V$ fusion protein $\left(100 \mu \mathrm{g} / \mathrm{ml}, \mathrm{BEl}\right.$ Resources) for $72 \mathrm{~h}$ at $37^{\circ} \mathrm{C}$, and then BrdU (BD Bioscience, San Jose, CA) was added to a final concentration of $10 \mu \mathrm{M}$ during the last $18 \mathrm{~h}$ of incubation with the recombinant protein to be incorporated into the splenocytes ${ }^{57,58}$.

Subsequently, the BrdU-labeled splenocytes were surface stained for Tcell (CD3e-APC; eBioscience) and B-cell (CD19-eFluor450, ThermoFisher Scientific, Grand Island, NY) markers after blocking with anti-mouse CD16/ 32 antibodies (BioLegend, San Diego, CA). Cells were then permeabilized and treated with DNase to expose BrdU epitopes followed by anti-BrdUFITC and 7-AAD (7-amino-actinomycin D) staining by using BD Pharmingen FITC BrdU Flow Kit (San Jose, CA). The splenocytes were then subjected to flow cytometry, and data analyzed as we previously described ${ }^{26}$. The percent of BrdU positive cells in CD3- and CD19-positive populations were calculated using FACSDiva software. The gating strategy for flow cytometry is provided in Supplementary Fig. 3.
For in vivo cell proliferation measurements, mice were injected (i.p.) with $1 \mathrm{mg}$ of BrdU at $24 \mathrm{~h}$ intervals during the first 3 days of infection with $Y$. pestis-lux CO92, and spleens were excised $1 \mathrm{~h}$ after the last BrdU injection $^{59}$. Splenocytes were then isolated and stained for CD3 and CD19 surface markers as well as for intracellular staining of BrdU as described above.

To assess cytokine production, the splenocytes on the duplicate plates from both ex vivo and in vivo experiments (as described above) were simulated with $\mathrm{rF} 1-\mathrm{V}(100 \mu \mathrm{g} / \mathrm{ml})$ for $72 \mathrm{~h}$ at $37^{\circ} \mathrm{C}$. Cell supernatants were then collected, and cytokines measured using Bio-Plex Pro Mouse Cytokine 23-plex Assay (Biorad Laboratories, Hercules, CA) following the manufacturer's standard protocol.

\section{T-cell phenotypes}

The splenocytes from duplicate plates from both ex vivo and in vivo experiments (as described above) were treated with ionomycin (750 ng/ $\mathrm{mL}$ ), PMA (phorbol 12-myristate 13-acetate) $(50 \mathrm{ng} / \mathrm{mL})$, and Brefeldin A $(5 \mu \mathrm{g} / \mathrm{mL})$ for $5 \mathrm{~h}$ at $37^{\circ} \mathrm{C}$ in a $5 \% \mathrm{CO}_{2}$ incubator. Splenocytes were then blocked with anti-mouse CD16/32 antibodies (BioLegend) followed by staining with Fixable Viability Dye eFluor ${ }^{\mathrm{TM}} 506$ (eBioscience) and APC antimouse CD3e (eBioscience), PE/Dazzle 594 anti-mouse CD4 (BioLegend), FITC anti-mouse CD8 (BioLegend) for CD3, CD4, and CD8 T-cell surface markers, respectively. Cells were then permeabilized for intracellular staining with PerCP/Cy5.5 anti-mouse IFNY (BioLegend) and analyzed by flow cytometry.

\section{Bronchoalveolar lavage and IgA production}

Twenty-eight days after $Y$. pestis CO92-lux infection, bronchoalveolar lavage fluids (BALF) were obtained from the surviving animals as well as from uninfected control mice to assess IgA antibody titers. We obtained BALF following the protocol as previously described with slight modifications ${ }^{60}$. Briefly, the salivary glands were dissected to expose the trachea from euthanized mice ( $n=5 /$ group). A small incision was made on the ventral face of the trachea and a blunt $26 \mathrm{G}$ needle was inserted into the trachea and secured by tying the trachea around the catheter using the floss placed underneath the trachea. An aliquot $(600 \mu \mathrm{L})$ of PBS was loaded into a $1 \mathrm{~mL}$ syringe and attached to the needle. Next, the solution was injected and then aspirated three times in and out of the lungs before being collected. The anti-F1-V IgA antibody titers were determined by ELISA with the HRP-conjugated goat anti-mouse IgA antibodies (1:8000, Southern Biotech) as the source of secondary antibodies, followed by color development as described in an earlier section. Similarly, we examined F1, LcrV, and YscF specific IgA in the serum of mice at the designated times post-immunization and post-challenge.

\section{Statistical analysis}

One-way or two-way analysis of variance (ANOVA) with Tukey's post-hoc test or the Student's $t$-test was used for data analysis. We used Kaplan-Meier with log-rank (Mantel-Cox) test for animal studies, and $P$ values of $\leq 0.05$ were considered significant for all the statistical tests used. The number of animals per group is described in each figure and two biological replicates were performed. All in vitro studies were performed in triplicates.

\section{Reporting summary}

Further information on research design is available in the Nature Research Reporting Summary linked to this article.

\section{DATA AVAILABILITY}

All data that this study is based upon are available from the corresponding authors upon request.

Received: 12 August 2020; Accepted: 14 December 2020; Published online: 29 January 2021

\section{REFERENCES}

1. Byard, R. W. A forensic evaluation of plague-a re-emerging infectious disease with biowarfare potential. Med. Sci. Law 60, 200-205 (2020). 
2. Shi, L. et al. Reemergence of human plague in Yunnan, China in 2016. PLoS ONE 13, e0198067 (2018).

3. Perry, R. D. \& Fetherston, J. D. Yersinia pestis-ētiologic agent of plague. Clin. Microbiol. Rev. 10, 35-66 (1997).

4. WHO (Regional Office for Africa). Plague outbreak situation reports. https://www. afro.who.int/health-topics/plague/plague-outbreak-situation-reports (2017).

5. Randremanana, R. et al. Epidemiological characteristics of an urban plague epidemic in Madagascar, August-November, 2017: an outbreak report. Lancet Infect. Dis. 19, 537-545 (2019).

6. Pechous, R. D., Sivaraman, V., Stasulli, N. M. \& Goldman, W. E. Pneumonic plague: the darker side of Yersinia pestis. Trends Microbiol. 24, 190-197 (2016).

7. Vallès, X. et al. Human plague: an old scourge that needs new answers. PLoS Negl. Trop. Dis. 14, 1-22 (2020).

8. Smiley, S. T. Current challenges in the development of vaccines for pneumonic plague. Expert Rev. Vaccines 7, 209-221 (2008).

9. Demeure, C. et al. Yersinia pestis and plague: an updated view on evolution, virulence determinants, immune subversion, vaccination and diagnostics. Microbes Infect. 21, 202-212 (2019).

10. Morris, S. R. Development of a recombinant vaccine against aerosolized plague. Vaccine 25, 3115-3117 (2007).

11. Fasciano, A. C., Shaban, L. \& Mecsas, J. Promises and challenges of the type three secretion system injectisome as an antivirulence target. EcoSal 8, 261-276 (2019).

12. Fellows, $P$. et al. Characterization of a cynomolgus macaque model of pneumonic plague for evaluation of vaccine efficacy. Clin. Vaccine Immunol. 22, 1070-1078 (2015).

13. Frey, S. E. et al. A phase I safety and immunogenicity dose escalation trial of plague vaccine, Flagellin/F1/N, in healthy adult volunteers (DMID 08-0066). Vaccine 35, 6759-6765 (2017)

14. Chu, K. et al. Immunogenicity and safety of subunit plague vaccine: a randomized phase 2a clinical trial. Hum. Vaccines Immunother. 12, 2334-2340 (2016).

15. Rosenzweig, J. A. et al. Progress on plague vaccine development. Appl. Microbiol. Biotechnol. 91, 265-286 (2011).

16. Russell, P. et al. A comparison of plague vaccine, USP and EV76 vaccine induced protection against Yersinia pestis in a murine model. Vaccine 13, 1551-1556 (1995).

17. Quenee, L. E. et al. Hereditary hemochromatosis restores the virulence of plague vaccine strains. J. Infect. Dis. 206, 1050-1058 (2012).

18. Meka-Mechenko, T. V. F1-negative natural Y. pestis Strains. Adv. Exp. Med. Biol. 529, 379-381 (2003).

19. Sha, J. et al. Characterization of an F1 deletion mutant of Yersinia pestis CO92, pathogenic role of $\mathrm{F} 1$ antigen in bubonic and pneumonic plague, and evaluation of sensitivity and specificity of F1 antigen capture-based dipsticks. J. Clin. Microbiol. 49, 1708-1715 (2011).

20. Daniel, C. et al. Polymorphism in the yersinia LcrV antigen enables immune escape from the protection conferred by an LcrV-secreting Lactococcus lactis in a pseudotuberculosis mouse model. Front. Immunol. 10, 1830 (2019).

21. Sun, W. \& Singh, A. K. Plague vaccine: recent progress and prospects. npj Vaccines 4, 11 (2019).

22. Bos, K. I. et al. Paleomicrobiology: diagnosis and evolution of ancient pathogens. Annu. Rev. Microbiol. 73, 639-666 (2019).

23. Achtman, M. et al. Yersinia pestis, the cause of plague, is a recently emerged clone of Yersinia pseudotuberculosis. Proc. Natl Acad. Sci. USA 96, 14043-14048 (1999).

24. Sha, J. et al. A Replication-Defective Human type 5 adenovirus-based trivalent vaccine confers complete protection against plague in mice and nonhuman primates. Clin. Vaccine Immunol. 23, 586-600 (2016).

25. WHO Workshop. Efficacy trials of Plague vaccines: endpoints, trial design, site selection. https://www.google.com/url?sa $=t \& r c t=j \& q=\& e s r c=s \&$ source $=$ web\&cd =\&ved $=2$ ahUKEwjUqsSx7p7uAhUHTawKHaMQBzUQFjAAegQIAhAC\&url=https\%3A\%2F\%2Fwww.who.int\%2Fblueprint $\% 2$ Fwhat $\%$ 2Fnormsstandards\%2FPlagueVxeval_FinalMeetingReport. pdf\&usg =AOvVaw2nPIcR6FgJA9HLKUnQgXUp (2018).

26. Tiner, B. L. et al. Immunisation of two rodent species with new live-attenuated mutants of Yersinia pestis CO92 induces protective long-term humoral- and cell-mediated immunity against pneumonic plague. npj Vaccines 1, 16020 (2016).

27. Boyer, J. L. et al. Protective immunity against a lethal respiratory Yersinia pestis challenge induced by $\mathrm{V}$ antigen or the $\mathrm{F} 1$ capsular antigen incorporated into adenovirus capsid. Hum. Gene Ther. 21, 891-901 (2010).

28. Teigler, J. E., lampietro, M. J. \& Barouch, D. H. Vaccination with adenovirus serotypes 35,26 , and 48 elicits higher levels of innate cytokine responses than adenovirus serotype 5 in Rhesus monkeys. J. Virol. 86, 9590-9598 (2012).

29. $\mathrm{Hu}, \mathrm{J}$. et al. One year immunogenicity and safety of subunit plague vaccine in Chinese healthy adults: an extended open-label study. Hum. Vaccine Immunother. 14, 1-5 (2018).
30. Heath, D. G. et al. Protection against experimental bubonic and pneumonic plague by a recombinant capsular F1-V antigen fusion protein vaccine. Vaccine 16, 1131-1137 (1998).

31. Williamson, E. D. et al. Recombinant $(F 1+$ V) vaccine protects cynomolgus macaques against pneumonic plague. Vaccine 29, 4771-4777 (2011).

32. Du, Y., Rosqvist, R. \& Forsberg, Å. Role of fraction 1 antigen of Yersinia pestis in inhibition of phagocytosis. Infect. Immun. 70, 1453-1460 (2002).

33. Demeure, C. E., Derbise, A. \& Carniel, E. Oral vaccination against plague using Yersinia pseudotuberculosis. Chem. Biol. Interact. 267, 89-95 (2017).

34. Matson, J. S., Durick, K. A., Bradley, D. S. \& Nilles, M. L. Immunization of mice with YscF provides protection from Yersinia pestis infections. BMC Microbiol. 5, 38 (2005).

35. Swietnicki, W., Powell, B. S. \& Goodin, J. Yersinia pestis Yop secretion protein F: purification, characterization, and protective efficacy against bubonic plague. Protein Expr. Purif. 42, 166-172 (2005).

36. Singh, A. K., Curtiss, R. \& Sun, W. A recombinant attenuated Yersinia pseudotuberculosis vaccine delivering a Y. pestis YopENt138-LcrV fusion elicits broad protection against plague and yersiniosis in mice. Infect. Immun. 87, e00296-19 (2019).

37. Gregg, K. A. et al. A lipid A-based TLR4 mimetic effectively adjuvants a Yersinia pestis $\mathrm{rF-V} 1$ subunit vaccine in a murine challenge model. Vaccine $\mathbf{3 6}, 4023-4031$ (2018).

38. Dinc, G., Pennington, J. M., Yolcu, E. S., Lawrenz, M. B. \& Shirwan, H. Improving the Th1 cellular efficacy of the lead Yersinia pestis rF1-V subunit vaccine using SA-4$1 \mathrm{BBL}$ as a novel adjuvant. Vaccine 32, 5035-5040 (2014).

39. Abbas, A. K., Murphy, K. M. \& Sher, A. Functional diversity of helper T lymphocytes. Nature 383, 787-793 (1996).

40. Rostamian, M., Sohrabi, S., Kavosifard, H. \& Niknam, H. M. Lower levels of IgG1 in comparison with $\operatorname{lgG} 2 \mathrm{a}$ are associated with protective immunity against Leishmania tropica infection in BALB/c mice. J. Microbiol. Immunol. Infect. 50, 160-166 (2017).

41. Zhang, Y. et al. IgG1 is required for optimal protection after immunization with the purified porin OmpD from Salmonella Typhimurium. J. Immunol. 199, 4103-4109 (2017).

42. Bowen, W. et al. Robust Th1 cellular and humoral responses generated by the Yersinia pestis $\mathrm{rF1}-\mathrm{V}$ subunit vaccine formulated to contain an agonist of the CD137 pathway do not translate into increased protection against pneumonic plague. Vaccine 37, 5708-5716 (2019).

43. Patterson, B. K. et al. CCR5 inhibition in critical COVID-19 patients decreases inflammatory cytokines, increases CD8 T-cells, and decreases SARS-CoV2 RNA in plasma by day 14. Int. J. Infect. Dis. 103, 25-32 (2021).

44. Dutta, S. \& Sengupta, P. Men and mice: relating their ages. Life Sci. 152, 244-248 (2016).

45. Anderson, G. W., Heath, D. G., Bolt, C. R., Welkos, S. L. \& Friedlander, A. M. Shortand long-term efficacy of single-dose sub unit vaccines against Yersinia pestis in mice. Am. J. Trop. Med. Hyg. 58, 793-799 (1998).

46. Wang, Z. et al. Long-term observation of subunit vaccine F1-rV270 against Yersinia pestis in mice. Clin. Vaccine Immunol. 17, 199-201 (2010).

47. Prame Kumar, K., Nicholls, A. J. \& Wong, C. H. Y. Partners in crime: neutrophils and monocytes/macrophages in inflammation and disease. Cell Tissue Res. 371, 551-565 (2018).

48. Comer, J. E. et al. Transcriptomic and innate immune responses to Yersinia pestis in the lymph node during bubonic plague. Infect. Immun. 78, 5086-5098 (2010).

49. Demeure, C. E. et al. Yersinia pestis and plague: an updated view on evolution, virulence determinants, immune subversion, vaccination, and diagnostics. Genes Immun. 20, 357-370 (2019).

50. Stasulli, N. M. et al. Spatially distinct neutrophil responses within the inflammatory lesions of pneumonic plague. mBio 6, e01530-15 (2015).

51. Gschwandtner, M., Derler, R. \& Midwood, K. S. More than just attractive: how CCL2 influences myeloid cell behavior beyond chemotaxis. Front. Immunol 10, 2759 (2019).

52. Bystry, R. S., Aluvihare, V., Welch, K. A., Kallikourdis, M. \& Betz, A. G. B cells and professional APCs recruit regulatory T cells via CCL4. Nat. Immunol. 2, 1126-1132 (2001).

53. Bhattacharya, P. et al. Dual role of GM-CSF as a pro-inflammatory and a regulatory cytokine: implications for immune therapy. J. Interferon Cytokine Res. 35, 585-599 (2015).

54. Steinman, R. M., Hawiger, D. \& Nussenzweig, M. C. Tolerogenic dendritic cells. Annu. Rev. Immunol. 21, 685-711 (2003).

55. Sha, J. et al. A non-invasive in vivo imaging system to study dissemination of bioluminescent Yersinia pestis CO92 in a mouse model of pneumonic plague. Microb. Pathog. 55, 39-50 (2013).

56. Tiner, B. L. et al. Combinational deletion of three membrane protein-encoding genes highly attenuates Yersinia pestis while retaining immunogenicity in a mouse model of pneumonic Plague. Infect. Immun. 83, 1318-1338 (2015). 
57. Penit, C. In vivo thymocyte maturation. BUdR labeling of cycling thymocytes and phenotypic analysis of their progeny support the single lineage model. J. Immunol 137, 2115-2121 (1986).

58. Endl, E., Steinbach, P., Knüchel, R. \& Hofstädter, F. Analysis of cell cycle-related Ki67 and p120 expression by flow cytometric BrdUrd-Hoechst/7AAD and immunolabeling technique. Cytometry 29, 233-241 (1997).

59. Rocha, B. et al. Accumulation of bromodeoxyuridine-labeled cells in central and peripheral lymphoid organs: minimal estimates of prodution and turnover rates of mature lymphocytes. Eur. J. Immunol. 20, 1697-1708 (1990).

60. van Hoecke, L., Job, E. R., Saelens, X. \& Roose, K. Bronchoalveolar lavage of murine lungs to analyze inflammatory cell infiltration. J. Vis. Exp. 123, https://www.jove. $\mathrm{com} / \mathrm{v} / 55398 /$ bronchoalveolar-lavage-murine-lungs-to-analyze-inflammatory-cell (2017).

\section{ACKNOWLEDGEMENTS}

P.B.K. was supported in part by Biodefense T32 Training Fellowship (T32-Al060549) awarded to A.K.C. We would like to thank rotation students Joseph Thiriot, Clint Haines, Galen Card, and Jacob Stockton for assistance with ELISA assays. We thank Dr. Yuejin Liang, Department of Microbiology and Immunology, in the analysis of the flow cytometry data. These studies were supported in part by funding from the $\mathrm{NIH}$ (Al153524 and Al071634) grants as well as UTMB Technology Commercialization Program funding awarded to A.K.C. Contributions of our company partner is greatly acknowledged.

\section{AUTHOR CONTRIBUTIONS}

P.B.K., J.S., V.L.M., and A.K.C. planned the experiments. P.B.K., J.S., and J.A.A. performed the experiments. P.B.K., J.S., J.A.A., V.L.M., and A.K.C. analyzed and discussed the data. P.B.K., J.S., and A.K.C. wrote the paper. P.B.K., J.S., V.L.M., and A.K.C. edited and revised the manuscript.

\section{COMPETING INTERESTS}

The authors declare no competing interests.

\section{ADDITIONAL INFORMATION}

Supplementary information The online version contains supplementary material available at https://doi.org/10.1038/s41541-020-00275-3.

Correspondence and requests for materials should be addressed to J.S. or A.K.C.

Reprints and permission information is available at http://www.nature.com/ reprints

Publisher's note Springer Nature remains neutral with regard to jurisdictional claims in published maps and institutional affiliations.

Open Access This article is licensed under a Creative Commons Attribution 4.0 International License, which permits use, sharing, adaptation, distribution and reproduction in any medium or format, as long as you give appropriate credit to the original author(s) and the source, provide a link to the Creative Commons license, and indicate if changes were made. The images or other third party material in this article are included in the article's Creative Commons license, unless indicated otherwise in a credit line to the material. If material is not included in the article's Creative Commons license and your intended use is not permitted by statutory regulation or exceeds the permitted use, you will need to obtain permission directly from the copyright holder. To view a copy of this license, visit http://creativecommons. org/licenses/by/4.0/.

(c) The Author(s) 2021 